\title{
A Limiting Case of Constant Counterion Electrochemical Potentials in the Membrane for Examining Concentration Polarization at Ion-exchange Membranes and Patches
}

Andriy Yaroshchuk, ${ }^{\mathrm{a}, \mathrm{b} *}$ Mykola Bondarenko, ${ }^{\mathrm{c}}$ Chao Tang, ${ }^{\mathrm{d}}$, and Merlin L. Bruening ${ }^{\mathrm{d}, \mathrm{e}^{*}}$

aCREA, pg.L.Companys 23, 08010 Barcelona, Spain

${ }^{b}$ Department of Chemical Engineering, Polytechnic University of Catalonia, av. Diagonal 647,08028

Barcelona, Spain

Institute of Bio-Colloid Chemistry, National Academy of Sciences of Ukraine, Vernadskiy ave.42, 03142, Kyiv, Ukraine

'Department of Chemical and Biomolecular Engineering, University of Notre Dame, Notre Dame, IN 46556, USA

${ }^{\mathrm{d} D e p a r t m e n t}$ of Chemistry, University of Notre Dame, Notre Dame, IN 46556, USA

*corresponding author. E-mail: andriy.yaroshchuk@upc.edu and mbruenin@nd.edu

\section{Supporting Information}

\section{Table of Contents}

S1. Description of Symbols.

S2. Origin of $\mathrm{Eq}(1)$, ion fluxes.

.S5

S3. Derivation of $\mathrm{Eq}(20)$, potential drop across the membrane (not including the boundary layers) under conditions of equal counterion electrochemical potentials

S4. Derivation of $\mathrm{Eq}(27)$, potential drop allowing for the resistance of the membrane and introduction of a third differential equation in the solution and in the membrane

S5. Derivation of Eqs(29-35), Concentration profiles under bi-ionic conditions with equal electrochemical potentials across the membrane

S6. Derivation of ion fluxes $(\mathrm{Eq}(36))$ under bi-ionic conditions and equal electrochemical potentials across the membrane 
S7. Derivation of expression for the bi-ionic potential drop, $(\mathrm{Eq}(37))$, under conditions of equal electrochemical potentials across the membrane

S8. Derivation of a first-order flux correction $(\mathrm{Eq}(38))$ to the limiting case of constant electrochemical potentials: Bi-ionic potentials.

S9. Derivation of a first-order potential difference correction $(\mathrm{Eq}(39))$ to the limiting case of constant electrochemical potentials: Bi-ionic potentials).

S10. Derivation of $\operatorname{Eqs}(42,43)$ for the flux and potential drop during current passage in the limiting case of constant electrochemical potentials across an ion-exchange membrane ........S18

S11. Derivation of $\mathrm{Eq}(45)$ for the flux of ion " 1 " during current passage: First-order correction to the limiting case of constant electrochemical potentials across an ion-exchange membrane

S12. Derivation of $\mathrm{Eq}(46)$ for the potential drop during current passage through an ionexchange membrane: First-order correction to the limiting case of constant electrochemical potentials across an ion-exchange membrane

S13. Numerical simulations of fluxes and potentials in bi-ionic systems .528

S14. Numerical simulations of current-induced concentration polarization...... .529

S15. Numerical simulations for modelling concentration profiles above a bipolar ion-exchange patch

\section{S1. Description of Symbols}

$A \quad \frac{2}{\left(\alpha^{2}+1\right)-2 \frac{C_{\Delta, O}}{C_{0}}\left(\alpha^{2}-1\right)} ;$ constant defined for convenience

c salt concentration in solution in the ion-exchange patch system

$\tilde{c} \quad c / c_{0}$ salt concentration divided by the bulk salt concentration in the ion-exchange patch system

$c_{0} \quad$ salt concentration in the bulk solution in the ion-exchange patch problem

$c_{i} \quad$ concentration of ion $i$ in the virtual solution for the boundary layer or in the numerical simulations, the real ion concentration in the boundary layer

$\bar{c}_{i} \quad$ concentration of ion $i$ in the real solution in the membrane or in the boundary layer

$c_{i 0} \quad$ concentration of ion $i$ in the left or right stirred solution 
$c_{X} \quad$ concentration of fixed charge in the membrane

$\bar{C} \quad$ sum of the mobile ion concentrations in the membrane

$C(\xi)$ or $C \quad$ sum of the concentrations of all ions in the virtual solution

$C_{0} \quad$ sum of the concentrations of all ions in the bulk stirred solution

$C_{\Delta} \quad c_{1}-c_{2} ;$ difference in the concentration of two counterions

$\bar{C}_{\Delta} \quad \bar{c}_{1}-\bar{c}_{2} ;$ difference in the real counterion concentrations in the membrane

$C_{\Delta, l} \quad c_{1}-c_{2}$ at the left side of the left boundary layer

$C_{\Delta, r} \quad c_{1}-c_{2}$ at the right side of the right boundary layer

$C_{\Delta, o} \quad c_{1}-c_{2}$ in the bulk solution

$D_{i} \quad$ ion diffusion coefficient in solution

$\bar{D}_{i} \quad$ diffusion coefficient of ion $i$ in the membrane, normalized by the boundary layer thickness

$E_{0} \quad$ constant bulk electric field defined in the ion-exchange patch system

$f \quad \frac{c_{10}}{c_{10}+c_{20}}$

F $\quad$ Faraday's constant

$g(\xi, \eta)$ function defined in $\mathrm{Eq}(62)$ for solving the Laplace equation

$i \quad$ index representing a specific ion

$j_{i} \quad$ flux of ion $i$

$j^{(1)} \quad$ small correction to the flux of ion " 1 " given in $\mathrm{Eq}(48)$

I current density

$\tilde{I} \quad$ current density divided by Faraday's constant

$\tilde{I}_{\text {lim }} \quad$ limiting current density divided by Faraday's constant

$\check{I} \quad \frac{\tilde{I}}{C_{0} \sqrt{P_{1} P_{2}}} ;$ dimensionless current

$K_{c} \quad$ integration constant in $\mathrm{Eq}(63)$

$K_{\varphi} \quad$ integration constant in $\mathrm{Eq}(64)$

$l \quad$ half width of an ion-exchange patch 
$L \quad$ Half thickness of the membrane divided by the boundary layer thickness

$P_{i} \quad$ boundary layer permeance to ion $i$, i.e. permeability divided by boundary layer thickness

$P_{i}^{*} \quad \Gamma_{i} D_{i} ;$ permeability of ion $i$

$P_{S} \quad$ salt permeance of the membrane

$R \quad$ gas constant

$S_{j} \quad \sum_{i} \frac{j_{i}}{P_{i}} ;$ sum of permeance-normalized fluxes

$\bar{S}_{j} \quad \frac{j_{1}}{\bar{D}_{1}}+\frac{j_{2}}{\bar{D}_{2}} ;$ sum of fluxes divided by the normalized diffusion coefficients in the membrane

$S_{Z} \quad \sum_{i} Z_{i} \cdot \frac{j_{i}}{P_{i}} ;$ sum of the ion charge multiplied by the permeance-normalized fluxes

$S_{\Delta} \quad \frac{j_{1}}{P_{1}}-\frac{j_{2}}{P_{2}} ;$ difference of permeance-normalized counterion fluxes

$\bar{S}_{\Delta} \quad \frac{j_{1}}{\bar{D}_{1}}-\frac{j_{2}}{\bar{D}_{2}}$; differences in counterion fluxes divided by normalized diffusion coefficients in the membrane

$t_{1} \quad \frac{P_{1} c_{10}}{P_{1} c_{10}+P_{2} c_{20}}$; transference number for counterion " 1 " in the boundary layer with an ideally permselective membrane

$t_{2} \frac{P_{2} c_{20}}{P_{1} c_{10}+P_{2} c_{20}}$; transference number for counterion " 2 " in the boundary layer with an ideally permselective membrane

T temperature

$x \quad$ coordinate for one-dimensional diffusion or a coordinate for the ion-exchange patch

$y \quad \frac{S_{j}}{C_{0}}$ or a coordinate for the ion-exchange patch

$y_{0} \quad$ the value of $\frac{S_{j}}{C_{0}}$ when $\beta=0$

$y_{1} \quad$ a constant for linear correction of $y$, i.e. $y=y_{0}+\beta y_{1}$

$Z_{i} \quad$ charge of ion $i$

$\alpha \quad \sqrt{\frac{P_{2}}{P_{1}}} ;$ square root of the ratio of permeances to counterion " 2 " and counterion " 1 " in the boundary layer 
$\bar{\alpha} \quad \sqrt{\frac{\bar{D}_{2}}{\bar{D}_{1}}}$; square root of the ratio of diffusion coefficients for counterion " 2 " and counterion " 1 " in the membrane

$\beta \quad \frac{L}{c_{X} / C_{0}} \cdot \sqrt{\frac{P_{1} P_{2}}{\bar{D}_{1} \bar{D}_{2}}} ;$ parameter defined for the linear correction

$\delta \quad$ boundary layer thickness

$\gamma_{i} \quad$ activity coefficient of ion $i$ in a virtual solution

$\bar{\gamma}_{i} \quad$ activity coefficient of ion $i$ in a real solution

$\Gamma_{i} \quad \frac{\bar{c}_{i}}{c_{i}} ;$ partition coefficient for ion $i$ between a real and virtual solution

$\eta \quad$ y-coordinate divided by the patch width

$\lambda \quad \frac{F E_{0} l}{2 R T}$ dimensionless voltage drop on the half width of the ion-exchange patch

$\bar{\mu}_{i} \quad$ electrochemical potential of ion $i$ for a real or virtual solution

$\mu_{i}^{o} \quad$ standard state chemical potential of ion $i$ in the virtual solution

$\bar{\mu}_{i}^{o} \quad$ real solution standard state chemical potential of ion $i$

$v_{i} \quad$ stoichiometric coefficient of ion $i$ in a salt

$\xi \quad x / \delta ; x$ coordinate divided by either the boundary layer thickness or the thickness of an ionexchange membrane patch

$\xi_{0} \quad$ any specificed $\mathrm{x}$-coordinate in the boundary layers divided by the boundary layer thickness

$\varphi \quad$ virtual electrostatic potential multiplied by $F / R T$

$\bar{\varphi} \quad$ real electrostatic potential multiplied by $F / R T$

$\phi \quad$ virtual electrostatic potential with dimensions

$\bar{\phi} \quad$ real electrostatic potential with dimensions

$\psi_{D} \quad$ Donnan potential at the membrane-boundary interface

\section{S2. Origin of Eq(1), ion fluxes}

In one dimension with no convection, $\mathrm{Eq}(\mathrm{S} 1)$ describes the transport of ion $i$, 
$j_{i}=-\frac{\bar{c}_{i} D_{i}}{R T} \frac{d \bar{\mu}_{i}}{d x}$

where $\bar{\mu}_{i}$ is the electrochemical potential of ion $i, D_{i}$ is the ion diffusion coefficient, $\bar{c}_{i}$ is the real concentration of this ion, $R$ is the gas constant and $T$ is temperature. Eq(S2) gives the electrochemical potential of the ion, where

$\bar{\mu}_{i}=\bar{\mu}_{i}^{o}+R T \ln \left(\bar{\gamma}_{i} \bar{c}_{i}\right)+Z_{i} F \bar{\phi}$

$\bar{\mu}_{i}^{o}$ is the standard-state electrochemical potential of the ion, $\bar{\gamma}_{i}$ is the activity coefficient, $Z_{i}$ is the ion charge and $\bar{\phi}$ is the real electrostatic potential.

Assuming that $\bar{\gamma}_{i}=1$, differentiating $\mathrm{Eq}(\mathrm{S} 2)$ and substituting into $\mathrm{Eq}(\mathrm{S} 1)$ yields the typical Nernst-Planck equation.

$j_{i}=-\bar{c}_{i} D_{i}\left(\frac{1}{\bar{c}_{i}} \frac{d \bar{c}_{i}}{d x}+\frac{Z_{i} F}{R T} \frac{d \bar{\phi}}{d x}\right)=-D_{i} \frac{d \overline{c_{i}}}{d x}-D_{i} \frac{\overline{c_{i} Z_{i} F}}{R T} \frac{d \bar{\phi}}{d x}$

Use of this equation requires both partition coefficients (to obtain boundary conditions for real concentrations) and diffusion coefficients. The use of virtual solutions, or solutions that could be in equilibrium with a given point in the membrane, simplifies the model in that it requires only a single permeability coefficient. For the virtual solution, we obtain

$\bar{\mu}_{i}=\mu_{i}^{o}+R T \ln \left(\gamma_{i} c_{i}\right)+Z_{i} F \phi$

where $c_{i}$ and $\phi$ are the virtual concentration and electrical potential, respectively. We define the partition coefficient

$\Gamma_{i}=\frac{\bar{c}_{i}}{c_{i}}$

Differentiation of $\mathrm{Eq}(\mathrm{S} 4)$, substitution into $\mathrm{Eq}(\mathrm{S} 1)$, and the use of $\mathrm{Eq}(\mathrm{S} 5)$ leads to

$j_{i}=-\Gamma_{i} D_{i} c_{i}\left(\frac{1}{c_{i}} \frac{d c_{i}}{d x}+\frac{Z_{i} F}{R T} \frac{d \phi}{d x}\right)=-P_{i}^{*}\left(\frac{d c_{i}}{d x}+c_{i} \frac{Z_{i} F}{R T} \frac{d \phi}{d x}\right)$

In this equation, we defined $P_{i}{ }^{*}=\Gamma_{i} D_{i}$, where $P_{i}{ }^{*}$ is the permeability to the ion.

We also define a dimensionless coordinate, $\xi$, where $\xi=x / \delta$ and $\delta$ is the boundary layer thickness. Additionally, we define a dimensionless electrostatic potential $\varphi=\phi \frac{F}{R T}$. This leads to

$j_{i}=-P_{i}\left(\frac{d c_{i}}{d \xi}+c_{i} Z_{i} \frac{d \varphi}{d \xi}\right)$

In this equation $P_{i}=P_{i}^{*} / \delta$, which is the membrane permeance.

S3. Derivation of $\mathrm{Eq}(20)$, potential drop across the membrane (not including the boundary layers) under conditions of equal counterion electrochemical potentials 
As noted in the text, equating ion electrochemical potentials in $\mathrm{Eq}(\mathrm{S} 4)$ for the ideal virtual solutions on the two faces of the membrane gives

$\ln \left(c_{1}(-0)\right)+\varphi(-0)=\ln \left(c_{1}(+0)\right)+\varphi(+0)$

$\ln \left(c_{2}(-0)\right)+\varphi(-0)=\ln \left(c_{2}(+0)\right)+\varphi(+0)$

where -0 and +0 denote the left and right surfaces of the infinitely thin membrane (see Fig. 1). Thus far, we solved the differential equations for the sum of ion concentrations and the potentials in the boundary layers (see $\mathrm{Eq}(6)$ and $\mathrm{Eq}(11)$ ), but we need to know the individual ion concentrations to substitute into $\mathrm{Eq}(\mathrm{S} 8)$ or $\mathrm{Eq}(\mathrm{S} 9)$ to solve for the potential drop across the infinitesimally thin membrane.

By subtracting $\mathrm{Eq}(\mathrm{S} 9)$ from $\mathrm{Eq}(\mathrm{S} 8)$, one obtains

$\ln \left(\frac{c_{1}(-0)}{c_{2}(-0)}\right)=\ln \left(\frac{c_{1}(+0)}{c_{2}(+0)}\right)$

We define the following variable

$C_{\Delta} \equiv c_{1}-c_{2}$

Using the definitions of $C_{\Delta}$ and $C$, and noting that for a solution containing only monovalent ions $c_{1}+$ $c_{2}-c_{3}=0$ (electroneutrality) in the boundary layers,

$c_{1} \equiv \frac{1}{4} \cdot\left(C+2 C_{\Delta}\right)$

$c_{2} \equiv \frac{1}{4} \cdot\left(C-2 C_{\Delta}\right)$

Substituting $\operatorname{Eqs}(\mathrm{S} 12, \mathrm{~S} 13)$ into $\mathrm{Eq}(\mathrm{S} 10)$ leads to

$\frac{C(-0)+2 C_{\Delta}(-0)}{C(-0)-2 C_{\Delta}(-0)}=\frac{C(+0)+2 C_{\Delta}(+0)}{C(+0)-2 C_{\Delta}(+0)}$

This equation transforms to

$\frac{C_{\Delta}(-0)}{C(-0)}=\frac{C_{\Delta}(+0)}{C(+0)}$

Substituting $\mathrm{Eq}(\mathrm{S} 12, \mathrm{~S} 13)$ into $\mathrm{Eq}(\mathrm{S} 8)$ leads to

$\varphi(-0)-\varphi(+0)=\ln \left(\frac{c_{1}(+0)}{c_{1}(-0)}\right)=\ln \left(\frac{C(+0)+2 C_{\Delta}(+0)}{C(-0)+2 C_{\Delta}(-0)}\right)=\ln \left(\frac{C(+0)\left(1+2 \frac{C_{\Delta}(+0)}{C(+0)}\right)}{C(-0)\left(1+2 \frac{C_{\Delta}(-0)}{C(-0)}\right)}\right)$

Finally, using $\mathrm{Eq}(15)$ gives

$\varphi(-0)-\varphi(+0)=\ln \left(\frac{C(+0)}{C(-0)}\right)=\ln \left(\frac{C_{0}+S_{j}}{C_{0}-S_{j}}\right)$ 
S4. Derivation of $\mathrm{Eq}(27)$, potential drop allowing for the resistance of the membrane and introduction of a third differential equation in the solution and in the membrane

Introduction of a third differential equation

The boundary layers.

To introduce a third differential equation, we define the following variable

$S_{\Delta} \equiv \frac{j_{1}}{P_{1}}-\frac{j_{2}}{P_{2}}$

where ions " 1 " and " 2 " have the same monovalent charge sign and are counterions of the ion-exchange membrane. Subtracting $\mathrm{Eq}(1)$ for ion " 2 " from $\mathrm{Eq}(1)$ for ion " 1 " yields

$-S_{\Delta}=\frac{d C_{\Delta}}{d \xi}+C_{\Delta} \cdot \frac{d \varphi}{d \xi}$

This is a first-order ordinary differential equation that can be solved in quadratures. The solution is

$C_{\Delta}(\xi)=\exp \left(\varphi\left(\xi_{0}\right)-\varphi(\xi)\right) \cdot\left[C_{\Delta}\left(\xi_{0}\right)-S_{\Delta} \cdot \int_{\xi_{0}}^{\xi} \exp \left(\varphi\left(\xi^{\prime}\right)-\varphi\left(\xi_{0}\right)\right) d \xi^{\prime}\right]$

By substituting $\mathrm{Eq}(11), \varphi(\xi)-\varphi\left(\xi_{0}\right)=\frac{s_{Z}}{S_{j}} \cdot \ln \left(\frac{C(\xi)}{C\left(\xi_{0}\right)}\right)$, we obtain

$C_{\Delta}(\xi)=\left(\frac{C(\xi)}{C\left(\xi_{0}\right)}\right)^{-\frac{S_{Z}}{S_{j}}}\left[C_{\Delta}\left(\xi_{0}\right)-S_{\Delta} \cdot \int_{\xi_{0}}^{\xi}\left(\frac{C\left(\xi^{\prime}\right)}{C\left(\xi_{0}\right)}\right)^{\frac{S_{Z}}{S_{j}}} d \xi^{\prime}\right]$

Substituting $\mathrm{Eq}(6), C(\xi)-C\left(\xi_{0}\right)=-S_{j} \cdot\left(\xi-\xi_{0}\right)$ into the numerator of the integral, after integration and transformation one obtains

$C_{\Delta}(\xi)=\left(C_{\Delta}\left(\xi_{0}\right)-\frac{s_{\Delta} \cdot C\left(\xi_{0}\right)}{S_{j}+S_{Z}}\right) \cdot\left(\frac{C(\xi)}{C\left(\xi_{0}\right)}\right)^{-\frac{S_{Z}}{S_{j}}}+\frac{S_{\Delta} \cdot C\left(\xi_{0}\right)}{S_{j}+S_{Z}} \cdot \frac{C(\xi)}{C\left(\xi_{0}\right)}$

With complete blockage of coions from the membrane and monovalent positive counterions

$j_{3}=0$

$S_{Z}=S_{j}$

Substituting $\mathrm{Eq}(\mathrm{S} 24)$ into $\mathrm{Eq}(\mathrm{S} 22)$ yields

$C_{\Delta}(\xi)=\left(C_{\Delta}\left(\xi_{0}\right)-\frac{s_{\Delta} \cdot C\left(\xi_{0}\right)}{2 S_{j}}\right) \cdot\left(\frac{C(\xi)}{C\left(\xi_{0}\right)}\right)^{-1}+\frac{S_{\Delta} \cdot C\left(\xi_{0}\right)}{2 S_{j}} \cdot \frac{C(\xi)}{C\left(\xi_{0}\right)}$

$\mathrm{Eq}(6)$ and $\mathrm{Eq}(\mathrm{S} 25)$ are relationships between the ion fluxes (contained in the constants $S_{\Delta}$ and $S_{j}$ ) and solution composition (given by $C(x)$ and $C_{\Delta}(x)$ since due to the electro-neutrality of virtual solutions only two virtual ion concentrations are independent. 


\section{Inside the membrane}

Next, we consider the membrane phase. Defining

$\bar{C}_{\Delta} \equiv \bar{c}_{1}-\bar{c}_{2}$ and $\bar{S}_{\Delta} \equiv \frac{j_{1}}{\bar{D}_{1}}-\frac{j_{2}}{\bar{D}_{2}}$

we obtain this first-order ordinary differential equation with constant coefficients for the difference of real counter-ion concentrations in the membrane.

$\frac{d \bar{C}_{\Delta}}{d \xi}-\bar{C}_{\Delta} \cdot \frac{\bar{S}_{j}}{c_{X}}+\bar{S}_{\Delta}=0$

(This equation relies on the identity that $\bar{C}_{\Delta} \cdot \frac{\bar{s}_{j}}{c_{X}}=-\bar{c}_{1} \frac{d \bar{\varphi}}{d \xi}+\bar{c}_{2} \frac{d \bar{\varphi}}{d \xi}$ and stems from Eq(S3) with nondimensionalized coordinate and potential and complete coion exclusion. We took the difference of the modified $\mathrm{Eq}(\mathrm{S} 3)$ for the two counterions.) $\mathrm{Eq}(\mathrm{S} 27)$ has this exponential solution that relates the function $\bar{C}_{\Delta}$ at two arbitrary points inside the membrane:

$\bar{C}_{\Delta}\left(\xi^{\prime}\right)=\bar{C}_{\Delta}(\xi) \cdot \exp \left(\frac{\bar{s}_{j}}{c_{X}} \cdot\left(\xi^{\prime}-\xi\right)\right)+c_{X} \cdot \frac{\bar{s}_{\Delta}}{\bar{s}_{j}} \cdot\left(1-\exp \left(\frac{\bar{s}_{j}}{c_{X}} \cdot\left(\xi^{\prime}-\xi\right)\right)\right)$

Next, we apply the boundary conditions of known solution compositions in the perfectly-stirred reservoirs and of Donnan equilibria at the membrane surfaces. Virtual concentrations just outside a membrane surface are related to the real concentrations just on the other side of this interface (inside the membrane) via exponentials of the Donnan potential.

Therefore, at the membrane surfaces

$\left(c_{1}+c_{2}\right) \cdot \exp \left(-\psi_{D}\right)=\bar{c}_{1}+\bar{c}_{2}=c_{X}$

where $\psi_{D}$ is the Donnan potential. Since, just outside the membrane, $c_{1}+c_{2}=\frac{C}{2}$

$\exp \left(-\psi_{D}\right)=\frac{2 c_{X}}{C}$

Accordingly,

$\bar{C}_{\Delta}($ membrane boundary $)=C_{\Delta}($ solution boundary $) \cdot \exp \left(-\psi_{D}\right)=C_{\Delta} \frac{2 c_{X}}{C}$

In particular, at the left and right membrane surfaces

$\bar{C}_{\Delta}(-L+0)=C_{\Delta}(-L-0) \cdot \frac{2 c_{X}}{C(-L-0)}$

$\bar{C}_{\Delta}(L-0)=C_{\Delta}(L+0) \cdot \frac{2 c_{X}}{C(L+0)}$

where $L$ is the membrane half-thickness scaled on the boundary-layer thickness. At the same time, using $\xi^{\prime}=L$ and $\xi=-L$ in $\mathrm{Eq}(\mathrm{S} 28)$ gives 
$\bar{C}_{\Delta}(L-0)=\bar{C}_{\Delta}(-L+0) \cdot \exp \left(\frac{2 L \bar{S}_{j}}{c_{X}}\right)+c_{X} \cdot \frac{\bar{s}_{\Delta}}{\bar{s}_{j}} \cdot\left(1-\exp \left(\frac{2 L \bar{S}_{j}}{c_{X}}\right)\right)$

By substituting $\operatorname{Eqs}(\mathrm{S} 32, \mathrm{~S} 33)$ into $\mathrm{Eq}(\mathrm{S} 34)$, we obtain

$\frac{C_{\Delta}(L+0)}{C(L+0)}=\frac{C_{\Delta}(-L-0)}{C(-L-0)} \cdot \exp \left(\frac{2 L \bar{S}_{j}}{c_{X}}\right)+\frac{\bar{S}_{\Delta}}{2 \bar{S}_{j}} \cdot\left(1-\exp \left(\frac{2 L \bar{S}_{j}}{c_{X}}\right)\right)$

When $\frac{2 L \bar{S}_{j}}{c_{X}} \rightarrow 0, \mathrm{Eq}(\mathrm{S} 35)$ becomes $\mathrm{Eq}(\mathrm{S} 15)$, consistent with the limiting case of zero differences of electrochemical potentials of counterions that should occur for very thin (small $L$ ), highly charged (large $c_{X}$ ) and permeable (small $\bar{S}_{j}$ ) membranes.

The derivation of Eqs(12-13) and Eq(S25) is not specific to the way we treat the membrane. Substituting coordinates into these three equations gives

$C(-L-0)=C_{0}-S_{j}$

$C(L+0)=C_{0}+S_{j}$

$C_{\Delta}(-L-0)=\left(C_{\Delta}(-L-1)-\frac{S_{\Delta} \cdot C_{0}}{2 S_{j}}\right) \cdot\left(\frac{C_{0}}{C_{0}-S_{j}}\right)+\frac{S_{\Delta}\left(C_{0}-S_{j}\right)}{2 S_{j}}$

$C_{\Delta}(L+0)=\left(C_{\Delta}(L+1)-\frac{S_{\Delta} \cdot C_{0}}{2 S_{j}}\right) \cdot\left(\frac{C_{0}}{C_{0}+S_{j}}\right)+\frac{S_{\Delta} \cdot\left(C_{0}+S_{j}\right)}{2 S_{j}}$

Note that $-L-0$ and $L+0$ correspond to positions in solution just to the left and right (see Fig. 2) of the membrane, respectively. Substituting Eqs(S36-S39) into Eq(S35) with rearrangements and noting the definition of the sinh function gives

$\left(\frac{C_{\Delta, l}}{C_{0}}-\frac{S_{\Delta}}{2 S_{j}}\right) \cdot\left(\frac{C_{0}}{C_{0}-S_{j}}\right)^{2} \cdot \exp \left(\frac{L \bar{S}_{j}}{C_{X}}\right)-\left(\frac{C_{\Delta, r}}{C_{0}}-\frac{S_{\Delta}}{2 S_{j}}\right) \cdot\left(\frac{C_{0}}{C_{0}+S_{j}}\right)^{2} \cdot \exp \left(-\frac{L \bar{S}_{j}}{C_{X}}\right)=-\left(\frac{S_{\Delta}}{S_{j}}-\frac{\bar{S}_{\Delta}}{\bar{S}_{j}}\right) \cdot \sinh \left(\frac{L \bar{S}_{j}}{c_{X}}\right)$

where

$C_{\Delta, l} \equiv C_{\Delta}(-L-1)$

$C_{\Delta, r} \equiv C_{\Delta}(L+1)$

The voltage drop across the system when the membrane has a finite thickness

Now we consider the entire system (boundary layers plus membrane). Eqs $(14,15)$ still apply (repeated below), but the values of the parameter $S_{j}$ will be different from the limiting case of zero electrochemical-potential differences and the coordinates will be different.

$\varphi(-1)-\varphi(-0)=\frac{S_{Z}}{S_{j}} \cdot \ln \left(\frac{C_{o}}{C_{o}-S_{j}}\right) \quad$ (left boundary layer) 
$\varphi(+0)-\varphi(+1)=\frac{S_{Z}}{S_{j}} \cdot \ln \left(\frac{C_{0}+S_{j}}{C_{0}}\right) \quad$ (right boundary layer)

If the membrane has a finite thickness, the left hand side of $\mathrm{Eq}(14)$ will represent $\varphi(-1-L)-$ $\varphi(-0-L)$. Similarly the left hand side of $\mathrm{Eq}(15)$ will represent $(\varphi(L+0)-\varphi(+1+L)$.

Substituting the coordinates into $\mathrm{Eq}(26)$ gives the difference of the real electrostatic potentials at the two interior sides of the membrane.

$\bar{\varphi}(-L+0)-\bar{\varphi}(L-0)=\frac{2 L \bar{S}_{j}}{c_{X}}$

Additionally the total potential drop between the two boundary layers should also include the difference between the two Donnan potentials (defined as the membrane potential minus the solution potential in both cases). Using $\mathrm{Eq}(\mathrm{S} 30)$

$\psi_{D}(-L)-\psi_{D}(L)=\ln \left(\frac{C(-0-L)}{C(L+0)}\right) \equiv \ln \left(\frac{C_{0}-S_{j}}{C_{0}+S_{j}}\right)$

Summing $\mathrm{Eq}(\mathrm{S} 43)$ and $\mathrm{Eq}(\mathrm{S} 44)$ gives the total potential drop between the boundary layers.

$\varphi(-L+0)-\varphi(L-0) \equiv \bar{\varphi}(-L+0)-\bar{\varphi}(L-0)-\left(\psi_{D}(-L)-\psi_{D}(L)\right)=\frac{2 L \bar{S}_{j}}{c_{X}}+\ln \left(\frac{C_{0}+S_{j}}{C_{0}-S_{j}}\right)$

Finally, adding up the virtual-potential drops across the boundary layers (Eq(17) and the membrane $(\mathrm{Eq}(\mathrm{S} 45))$, for the total electrostatic-potential difference we obtain

$\varphi(-1-L)-\varphi(1+L)=2\left[\frac{L \bar{S}_{j}}{c_{X}}+\ln \left(\frac{C_{0}+S_{j}}{C_{0}-S_{j}}\right)\right]$

Note that $\mathrm{Eq}(\mathrm{S} 46)$ assumes $\frac{S_{Z}}{S_{j}}=1$.

S5. Derivation of Eqs(29-35), Concentration profiles under bi-ionic conditions with equal electrochemical potentials across the membrane

In the bi-ionic configuration with different 1:1 salts on each side of the membrane, but at the same concentration $C_{0}$, the following apply with complete coion exclusion. (Ion " 1 " is not present in the right perfectly stirred layer and ion " 2 " is not present in the left perfectly stirred layer.)

$C_{\Delta, l}=C_{0} / 2$

$C_{\Delta, r}=-C_{0} / 2$

$j_{2}=-j_{1}$

Based on the definitions of $S_{j}$ and $S_{\Delta}$,

$\frac{S_{\Delta}}{S_{j}}=\frac{P_{2}+P_{1}}{P_{2}-P_{1}}$ 
Starting from $\mathrm{Eq}(\mathrm{S} 25)$ (repeated here for convenience),

$C_{\Delta}(\xi)=\left(C_{\Delta}\left(\xi_{0}\right)-\frac{s_{\Delta} \cdot C\left(\xi_{0}\right)}{2 S_{j}}\right) \cdot\left(\frac{C(\xi)}{C\left(\xi_{0}\right)}\right)^{-1}+\frac{s_{\Delta} \cdot C\left(\xi_{0}\right)}{2 S_{j}} \cdot \frac{C(\xi)}{C\left(\xi_{0}\right)}$

for the infinitesimally thin membrane with substitution of $\operatorname{Eqs}(12,13)$ we obtain

$C_{\Delta}(-0)=\left(C_{\Delta, l}-\frac{S_{\Delta} \cdot C_{0}}{2 S_{j}}\right) \cdot\left(\frac{C_{0}}{C_{0}-S_{j}}\right)+\frac{S_{\Delta} \cdot\left(C_{0}-S_{j}\right)}{2 S_{j}}$

$C_{\Delta}(+0)=\left(C_{\Delta, r}-\frac{S_{\Delta} \cdot C_{0}}{2 S_{j}}\right) \cdot\left(\frac{C_{0}}{C_{0}+S_{j}}\right)+\frac{S_{\Delta} \cdot\left(C_{0}+S_{j}\right)}{2 S_{j}}$

Inserting $\operatorname{Eqs}(\mathrm{S} 51, \mathrm{~S} 52)$ and $\operatorname{Eqs}(12,13)$ into $\mathrm{Eq}(\mathrm{S} 15)$ gives

$\left(C_{\Delta, l}-\frac{S_{\Delta} \cdot C_{0}}{2 S_{j}}\right) \cdot \frac{1}{\left(C_{0}-S_{j}\right)^{2}}=\left(C_{\Delta, r}-\frac{S_{\Delta} \cdot C_{0}}{2 S_{j}}\right) \cdot \frac{1}{\left(C_{0}+S_{j}\right)^{2}}$

Substituting $\mathrm{Eq}(\mathrm{S} 47, \mathrm{~S} 48$, and $\mathrm{S} 50)$ into $\mathrm{Eq}(\mathrm{S} 53)$, with appropriate rearrangements, we obtain

$\frac{S_{j}}{C_{0}}=\frac{\alpha-1}{\alpha+1}$

where

$\alpha^{2} \equiv P_{2} / P_{1}$

In the left boundary layer, $\mathrm{Eq}(6) C(\xi)-C\left(\xi_{0}\right)=-S_{j} \cdot\left(\xi-\xi_{0}\right)$ leads to

$\frac{C(\xi)}{C_{0}}=1-\frac{S_{j}}{C_{0}}(\xi+1)$

Substituting $\mathrm{Eq}(\mathrm{S} 54)$ into $\mathrm{Eq}(\mathrm{S} 56)$ gives

$\frac{C(\xi)}{C_{0}}=1-\left(\frac{\alpha-1}{\alpha+1}\right)(1+\xi)$

Starting from $\mathrm{Eq}(\mathrm{S} 12), c_{1}=\frac{1}{4} \cdot\left(C+2 C_{\Delta}\right)$ and inserting the expression for $C_{\Delta}(\xi)$ in $\mathrm{Eq}(\mathrm{S} 25)$ leads to

$c_{1} \equiv \frac{1}{4} \cdot\left(C(\xi)+\left(C_{0}-\frac{s_{\Delta} \cdot C_{0}}{s_{j}}\right)\left(\frac{C(\xi)}{C_{0}}\right)^{-1}+\frac{s_{\Delta} \cdot C(\xi)}{S_{j}}\right)$

Substituting $\mathrm{Eq}(\mathrm{S} 50)$ and appropriate transformations leads to

$c_{1}(\xi)=\frac{C_{0}}{2} \cdot \frac{\alpha^{2} \cdot \frac{C(\xi)}{C_{0}}-\frac{C_{0}}{C(\xi)}}{\alpha^{2}-1}$

Similarly,

$c_{2}(\xi)=\frac{C_{0}}{2} \cdot \frac{\frac{C_{0}}{C(\xi)}-\frac{C(\xi)}{C_{0}}}{\alpha^{2}-1}$ 
Derivation of the concentration profiles in the boundary layer on the right side of the membrane follows similarly.

S6. Derivation of ion fluxes (Eq(36)) under bi-ionic conditions and equal electrochemical potentials across the membrane

For complete coion exclusion $j_{1}=-j_{2}$ and

$S_{j} \equiv \sum_{i} \frac{j_{i}}{P_{i}}=\frac{j_{1}}{P_{1}}-\frac{j_{1}}{P_{2}}$

Dividing this expression by $C_{0}$ and substituting $\mathrm{Eq}(\mathrm{S} 54)$ along with appropriate transformations leads to

$j_{1}=\frac{C_{0}}{\left(\frac{1}{\sqrt{P_{1}}}+\frac{1}{\sqrt{P_{2}}}\right)^{2}}$

S7. Derivation of an expression for the bi-ionic potential drop, $(\mathrm{Eq}(37))$, under conditions of equal electrochemical potentials across the membrane

Starting from $\mathrm{Eq}(21)$ and substituting the expression for $\frac{S_{j}}{C_{0}}$ in $\mathrm{Eq}(\mathrm{S} 54)$ leads to

$\varphi(-1)-\varphi(1)=2 \ln \left(\frac{C_{0}+S_{j}}{C_{0}-S_{j}}\right)=2 \ln \left(\frac{1+\frac{S_{j}}{C_{0}}}{1-\frac{S_{j}}{C_{0}}}\right)=2 \ln \left(\frac{1+\frac{\alpha-1}{\alpha+1}}{1-\frac{\alpha-1}{\alpha+1}}\right)$

Rearrangement gives

$\varphi(-1)-\varphi(1)=2 \ln \left(\frac{\frac{2 \alpha}{\alpha+1}}{\frac{2}{\alpha+1}}\right)=2 \ln (\alpha)=\ln \left(\alpha^{2}\right)=\ln \left(\frac{P_{2}}{P_{1}}\right)$

S8. Derivation of a first-order flux correction (Eq(38)) to the limiting case of constant electrochemical potentials: Bi-ionic potentials

This derivation starts with $\mathrm{Eq}(\mathrm{S} 40)$, which we repeat hear for convenience.

$\left(\frac{C_{\Delta, l}}{C_{0}}-\frac{S_{\Delta}}{2 S_{j}}\right) \cdot\left(\frac{C_{0}}{C_{0}-S_{j}}\right)^{2} \cdot \exp \left(\frac{L \bar{S}_{j}}{c_{X}}\right)-\left(\frac{C_{\Delta, r}}{C_{0}}-\frac{S_{\Delta}}{2 S_{j}}\right) \cdot\left(\frac{C_{0}}{C_{0}+S_{j}}\right)^{2} \cdot \exp \left(-\frac{L \bar{S}_{j}}{c_{X}}\right)=-\left(\frac{S_{\Delta}}{S_{j}}-\frac{\bar{S}_{\Delta}}{\bar{S}_{j}}\right) \cdot \sinh \left(\frac{L \bar{S}_{j}}{c_{X}}\right)(S 40$

We assume that the terms $\frac{L \bar{s}_{j}}{c_{X}}$ is small. This leads to the following linear approximations

$\sinh \left(\frac{L \bar{S}_{j}}{c_{X}}\right)=\frac{L \bar{s}_{j}}{c_{X}} ; \exp \left(\frac{L \bar{S}_{j}}{c_{X}}\right)=1+\frac{L \bar{S}_{j}}{c_{X}} ; \exp \left(-\frac{L \bar{S}_{j}}{c_{X}}\right)=1-\frac{L \bar{S}_{j}}{c_{X}}$

Inserting these approximations along with $\frac{C_{\Delta, l}}{C_{0}}=-\frac{C_{\Delta, r}}{C_{0}}=\frac{1}{2}$ (monovalent salts with only one salt in each stirred solution) leads to 


$$
\begin{aligned}
& \left(1-\frac{S_{\Delta}}{S_{j}}\right) \cdot\left(\frac{C_{0}}{C_{0}-S_{j}}\right)^{2}+\frac{L \bar{S}_{j}}{c_{X}}\left(1-\frac{S_{\Delta}}{S_{j}}\right) \cdot\left(\frac{C_{0}}{C_{0}-S_{j}}\right)^{2}-\left(-1-\frac{S_{\Delta}}{S_{j}}\right) \cdot\left(\frac{C_{0}}{C_{0}+S_{j}}\right)^{2}+\frac{L \bar{S}_{j}}{c_{X}}\left(-1-\frac{S_{\Delta}}{S_{j}}\right) \cdot\left(\frac{C_{0}}{C_{0}+S_{j}}\right)^{2}+ \\
& 2\left(\frac{S_{\Delta}}{S_{j}}-\frac{\bar{S}_{\Delta}}{\bar{S}_{j}}\right) \cdot \frac{L \bar{S}_{j}}{c_{X}}=0
\end{aligned}
$$

Rearranging yields

$$
\begin{aligned}
& \left(1-\frac{S_{\Delta}}{S_{j}}\right) \cdot\left(\frac{C_{0}}{C_{0}-S_{j}}\right)^{2}+\left(1+\frac{S_{\Delta}}{S_{j}}\right) \cdot\left(\frac{C_{0}}{C_{0}+S_{j}}\right)^{2}+\frac{L \bar{S}_{j}}{c_{X}}\left\{\left(1-\frac{S_{\Delta}}{S_{j}}\right) \cdot\left(\frac{C_{0}}{C_{0}-S_{j}}\right)^{2}-\left(1+\frac{S_{\Delta}}{S_{j}}\right) \cdot\left(\frac{C_{0}}{C_{0}+S_{j}}\right)^{2}+\right. \\
& \left.2\left(\frac{S_{\Delta}}{S_{j}}-\frac{\bar{s}_{\Delta}}{\overline{S_{j}}}\right)\right\}=0
\end{aligned}
$$

This equation relates sums and differences of fluxes to permeances, diffusion coefficients, and the bulk sum of concentration. Our task is to make the relationship between a specific ion flux and the permeances and normalized diffusion coefficients explicit. Based on their definitions, we also have the following expressions

$\frac{S_{\Delta}}{S_{j}}=\frac{P_{2}+P_{1}}{P_{2}-P_{1}}=\frac{\alpha^{2}+1}{\alpha^{2}-1} ; \alpha, \bar{\alpha} \equiv \sqrt{\frac{P_{2}}{P_{1}}}, \sqrt{\frac{\bar{D}_{2}}{\bar{D}_{1}}} ; \beta \equiv \frac{L}{c_{X} / C_{0}} \cdot \sqrt{\frac{P_{1} P_{2}}{\bar{D}_{1} \bar{D}_{2}}} ; y \equiv \frac{S_{j}}{C_{0}}$

Additionally, from the definitions of $\bar{S}_{j}$ and $S_{j}$, and the assumption of steady state and coion exclusion with $j_{2}=-j_{1}$

$\frac{\bar{S}_{j}}{C_{0}}=\frac{S_{j}}{C_{0}} \cdot \frac{\frac{1}{\bar{D}_{1}}-\frac{1}{\bar{D}_{2}}}{\frac{1}{P_{1}}-\frac{1}{P_{2}}}=y \cdot \frac{P_{2}}{\bar{D}_{2}} \cdot\left(\frac{\bar{\alpha}^{2}-1}{\alpha^{2}-1}\right)=y \cdot\left(\frac{\bar{\alpha}^{2}-1}{\alpha^{2}-1}\right) \sqrt{\frac{P_{2}}{\bar{D}_{2}}} \cdot \sqrt{\frac{\bar{D}_{1}}{P_{1}}} \sqrt{\frac{P_{1} P_{2}}{\bar{D}_{1} \bar{D}_{2}}}=y \cdot\left(\frac{\bar{\alpha}^{2}-1}{\alpha^{2}-1}\right) \frac{\alpha}{\bar{\alpha}} \sqrt{\frac{P_{1} P_{2}}{\bar{D}_{1} \bar{D}_{2}}} \equiv y \cdot\left(\frac{\bar{\alpha}-\frac{1}{\bar{\alpha}}}{\alpha-\frac{1}{\alpha}}\right) \sqrt{\frac{P_{1} P_{2}}{\bar{D}_{1} \bar{D}_{2}}}$

Moreover, the definitions of $\bar{S}_{\Delta}$ and $\bar{S}_{j}$ and $j_{2}=-j_{1}$ give

$\frac{\bar{S}_{\Delta}}{\bar{S}_{j}}=\frac{\frac{1}{\bar{D}_{1}}-\frac{1}{\bar{D}_{2}}}{\frac{1}{P_{1}}-\frac{1}{P_{2}}}=\frac{\bar{\alpha}^{2}+1}{\bar{\alpha}^{2}-1}$

$\mathrm{Eq}(\mathrm{S} 67)$ contains the term $\frac{L \bar{S}_{j}}{c_{X}}$. We can rewrite this term as

$\frac{L \bar{S}_{j}}{c_{X}} \equiv \frac{L}{c_{X} / C_{0}} \frac{\bar{S}_{j}}{C_{0}} \equiv \frac{L}{c_{X} / C_{0}} y \cdot\left(\frac{\bar{\alpha}-\frac{1}{\bar{\alpha}}}{\alpha-\frac{1}{\alpha}}\right) \sqrt{\frac{P_{1} P_{2}}{\bar{D}_{1} \bar{D}_{2}}} \equiv \beta y \cdot\left(\frac{\bar{\alpha}-\frac{1}{\bar{\alpha}}}{\alpha-\frac{1}{\alpha}}\right)$

Substituting Eqs(S68,S70,S71) into Eq(67) gives

$$
\begin{aligned}
& \left(1-\frac{\alpha^{2}+1}{\alpha^{2}-1}\right) \cdot\left(\frac{1}{1-y}\right)^{2}+\left(1+\frac{\alpha^{2}+1}{\alpha^{2}-1}\right) \cdot\left(\frac{1}{1+y}\right)^{2}+\left\{\left(1-\frac{\alpha^{2}+1}{\alpha^{2}-1}\right) \cdot\left(\frac{1}{1-y}\right)^{2}-\left(1+\frac{\alpha^{2}+1}{\alpha^{2}-1}\right) \cdot\left(\frac{1}{1+y}\right)^{2}+\right. \\
& \left.2\left(\frac{\alpha^{2}+1}{\alpha^{2}-1}-\frac{\bar{\alpha}^{2}+1}{\bar{\alpha}^{2}-1}\right)\right\} \cdot \beta y \cdot\left(\frac{\bar{\alpha}-\frac{1}{\bar{\alpha}}}{\alpha-\frac{1}{\alpha}}\right)=0
\end{aligned}
$$

The use of common denominators leads to 
$\left(\frac{-2}{\alpha^{2}-1}\right) \cdot\left(\frac{1}{1-y}\right)^{2}+\left(\frac{2 \alpha^{2}}{\alpha^{2}-1}\right) \cdot\left(\frac{1}{1+y}\right)^{2}+\left\{\left(\frac{-2}{\alpha^{2}-1}\right) \cdot\left(\frac{1}{1-y}\right)^{2}-\left(\frac{2 \alpha^{2}}{\alpha^{2}-1}\right) \cdot\left(\frac{1}{1+y}\right)^{2}+2\left(\frac{\alpha^{2}+1}{\alpha^{2}-1}-\frac{\bar{\alpha}^{2}+1}{\bar{\alpha}^{2}-1}\right)\right\} \cdot \beta y \cdot$ $\left(\frac{\bar{\alpha}-\frac{1}{\bar{\alpha}}}{\alpha-\frac{1}{\alpha}}\right)=0$

Noting that $\frac{\alpha^{2}+1}{\alpha^{2}-1}-\frac{\bar{\alpha}^{2}+1}{\bar{\alpha}^{2}-1}=\frac{\left(\alpha^{2}+1\right)\left(\bar{\alpha}^{2}-1\right)-\left(\alpha^{2}-1\right)\left(\bar{\alpha}^{2}+1\right)}{\left(\alpha^{2}-1\right)\left(\bar{\alpha}^{2}-1\right)}=\frac{2\left(\bar{\alpha}^{2}-\alpha^{2}\right)}{\left(\alpha^{2}-1\right)\left(\bar{\alpha}^{2}-1\right)}$, substituting this expression into $\mathrm{Eq}(\mathrm{S} 73)$ and multiplying both sides of $\mathrm{Eq}(\mathrm{S} 73)$ by $-\frac{\left(\alpha^{2}-1\right)}{2}$ leads to

$\left(\frac{1}{1-y}\right)^{2}-\alpha^{2} \cdot\left(\frac{1}{1+y}\right)^{2}+\left\{\left(\frac{1}{1-y}\right)^{2}+\alpha^{2} \cdot\left(\frac{1}{1+y}\right)^{2}-\frac{2\left(\bar{\alpha}^{2}-\alpha^{2}\right)}{\left(\bar{\alpha}^{2}-1\right)}\right\} \cdot \beta y \cdot\left(\frac{\bar{\alpha}-\frac{1}{\bar{\alpha}}}{\alpha-\frac{1}{\alpha}}\right)=0$

Multiplying both sides of the equation by $(1+y)^{2}$ yields

$\left(\frac{1+y}{1-y}\right)^{2}-\alpha^{2}+\left\{\left(\frac{1+y}{1-y}\right)^{2}+\alpha^{2}-(1+y)^{2} \cdot \frac{2\left(\bar{\alpha}^{2}-\alpha^{2}\right)}{\left(\bar{\alpha}^{2}-1\right)}\right\} \cdot \beta y \cdot\left(\frac{\bar{\alpha}-\frac{1}{\bar{\alpha}}}{\alpha-\frac{1}{\alpha}}\right)=0$

Next, we make assume a linear correction in the sum of the fluxes, $S_{j}$, due to the finite membrane thickness. We do this in the form

$y=\frac{s_{j}}{C_{0}}=y_{0}+\beta y_{1}$

In $\mathrm{Eq}(\mathrm{S} 76), y_{0}$ is the value of $\frac{S_{j}}{C_{0}}$ when $\beta=0$, and $y_{1}$ is a constant. Note that when $\beta=0$, we return to the limiting case. Substituting $\mathrm{Eq}(\mathrm{S} 76)$ into $\mathrm{Eq}(\mathrm{S} 75)$ and retaining only expressions with linear terms in $\beta$ leads to

$\left(\frac{1+y_{0}+\beta y_{1}}{1-y_{0}-\beta y_{1}}\right)^{2}-\alpha^{2}+\left\{\left(\frac{1+y_{0}}{1-y_{0}}\right)^{2}+\alpha^{2}-\left(1+y_{0}\right)^{2} \cdot \frac{2\left(\bar{\alpha}^{2}-\alpha^{2}\right)}{\left(\bar{\alpha}^{2}-1\right)}\right\} \cdot \beta y_{0} \cdot\left(\frac{\bar{\alpha}-\frac{1}{\bar{\alpha}}}{\alpha-\frac{1}{\alpha}}\right)=0$

Note that because the term in braces is multiplied by $\beta$, we discarded the corrections within the braces.

Substituting $y_{0}=\frac{\alpha-1}{\alpha+1}\left(\mathrm{Eq}(\mathrm{S} 54)\right.$ and $\frac{1+y_{0}}{1-y_{0}}=\frac{\frac{\alpha+1+\alpha-1}{\alpha+1}}{\frac{\alpha+1-\alpha+1}{\alpha+1}}=\frac{2 \alpha}{2}=\alpha$,

$\left(\frac{1+y_{0}+\beta y_{1}}{1-y_{0}-\beta y_{1}}\right)^{2}-\alpha^{2}+\left\{\alpha^{2}+\alpha^{2}-\left(1+\frac{\alpha-1}{\alpha+1}\right)^{2} \cdot \frac{2\left(\bar{\alpha}^{2}-\alpha^{2}\right)}{\left(\bar{\alpha}^{2}-1\right)}\right\} \cdot \beta \cdot\left(\frac{\alpha-1}{\alpha+1}\right) \cdot\left(\frac{\bar{\alpha}-\frac{1}{\bar{\alpha}}}{\alpha-\frac{1}{\alpha}}\right)=0$

Multiplying $\left(\frac{1+y_{0}+\beta y_{1}}{1-y_{0}-\beta y_{1}}\right)$ by $\left(\frac{1+y_{0}}{1-y_{0}}\right)\left(\frac{1-y_{0}}{1+y_{0}}\right)$ yields

$\left(\frac{1+y_{0}+\beta y_{1}}{1-y_{0}-\beta y_{1}}\right)=\frac{1+y_{0}}{1-y_{0}}\left(\frac{1+\frac{\beta y_{1}}{1+y_{0}}}{1-\frac{\beta y_{1}}{1-y_{0}}}\right)$

Reemembering that $\frac{1+y_{0}}{1-y_{0}}=\alpha$ (rearrangement of Eq(S54) leads to 
$\left(\frac{1+y_{0}+\beta y_{1}}{1-y_{0}-\beta y_{1}}\right)=\alpha\left(\frac{1+\frac{\beta y_{1}}{1+y_{0}}}{1-\frac{\beta y_{1}}{1-y_{0}}}\right)$ so $\left(\frac{1+y_{0}+\beta y_{1}}{1-y_{0}-\beta y_{1}}\right)^{2}=\alpha^{2}\left(\frac{1+\frac{\beta y_{1}}{1+y_{0}}}{1-\frac{\beta y_{1}}{1-y_{0}}}\right)^{2}$

If $\frac{\beta y_{1}}{1+y_{0}}$ is small $\left(\frac{1+\frac{\beta y_{1}}{1+y_{0}}}{1-\frac{\beta y_{1}}{1-y_{0}}}\right)^{2} \approx\left(1+\frac{2 \beta y_{1}}{1+y_{0}}\right)\left(1+\frac{2 \beta y_{1}}{1-y_{0}}\right)$. Thus, taking only linear terms

$\left(\frac{1+y_{0}+\beta y_{1}}{1-y_{0}-\beta y_{1}}\right)^{2}=\alpha^{2}\left(\frac{1+\frac{\beta y_{1}}{1+y_{0}}}{1-\frac{\beta y_{1}}{1-y_{0}}}\right)^{2} \approx \alpha^{2}+2 \alpha^{2} \beta y_{1}\left(\frac{1}{1+y_{0}}+\frac{1}{1-y_{0}}\right)=\alpha^{2}+\frac{4 \alpha^{2} \beta y_{1}}{1-y_{0}^{2}}$

Substituting for $y_{0}=\frac{\alpha-1}{\alpha+1}$ and rearranging gives

$\left(\frac{1+y_{0}+\beta y_{1}}{1-y_{0}-\beta y_{1}}\right)^{2}=\alpha^{2}\left[1+\left(\frac{4 \beta y_{1} \cdot(1+\alpha)^{2}}{(1+\alpha)^{2}-(1-\alpha)^{2}}\right)\right] \equiv \alpha^{2}\left[1+(1+\alpha)^{2}\left(\frac{\beta y_{1}}{\alpha}\right)\right]$

Subsituting $\mathrm{Eq}(\mathrm{S} 82)$ into $\mathrm{Eq}(\mathrm{S} 78)$ yields

$\alpha^{2}\left[1+(1+\alpha)^{2}\left(\frac{\beta y_{1}}{\alpha}\right)\right]-\alpha^{2}+\left\{2 \alpha^{2}-\left(\frac{2 \alpha}{\alpha+1}\right)^{2} \cdot \frac{2\left(\bar{\alpha}^{2}-\alpha^{2}\right)}{\left(\bar{\alpha}^{2}-1\right)}\right\} \cdot \beta \cdot\left(\frac{\alpha-1}{\alpha+1}\right) \cdot\left(\frac{\bar{\alpha}-\frac{1}{\bar{\alpha}}}{\alpha-\frac{1}{\alpha}}\right)=0$

This is identical to

$\beta\left\{(1+\alpha)^{2} \cdot y_{1}+2 \alpha \cdot\left[1-\left(\frac{2}{1+\alpha}\right)^{2} \cdot \frac{\left(\bar{\alpha}^{2}-\alpha^{2}\right)}{\left(\bar{\alpha}^{2}-1\right)}\right] \cdot\left(\frac{\bar{\alpha}-\frac{1}{\bar{\alpha}}}{\alpha-\frac{1}{\alpha}}\right) \cdot y_{0}\right\}=0$

Dividing by $\beta$ and applying significant transformations leads to

$$
(1+\alpha)^{2} \cdot y_{1}+\frac{2 \alpha^{2}}{\alpha^{2}-1} \cdot\left[\left(\bar{\alpha}-\frac{1}{\bar{\alpha}}\right)-\left(\frac{2}{\frac{1}{\sqrt{\alpha}}+\sqrt{\alpha}}\right)^{2} \cdot\left(\frac{\bar{\alpha}}{\alpha}-\frac{\alpha}{\bar{\alpha}}\right)\right] \cdot y_{0}=0
$$

Finally,

$y_{1}=-\frac{2 \alpha^{2}}{(1+\alpha)^{2}\left(\alpha^{2}-1\right)}\left[\left(\bar{\alpha}-\frac{1}{\bar{\alpha}}\right)-\left(\frac{2}{\frac{1}{\sqrt{\alpha}}+\sqrt{\alpha}}\right)^{2} \cdot\left(\frac{\bar{\alpha}}{\alpha}-\frac{\alpha}{\bar{\alpha}}\right)\right] \cdot y_{0}=-\frac{2 \alpha^{2}}{(1+\alpha)^{2}\left(\alpha^{2}-1\right)}\left[\left(\bar{\alpha}-\frac{1}{\bar{\alpha}}\right)-\left(\frac{2}{1+\alpha}\right)^{2}\right.$.

$\left.\left(\bar{\alpha}-\frac{\alpha^{2}}{\bar{\alpha}}\right)\right] \cdot y_{0}$

From the definition of $S_{j}=\frac{j_{1}}{P_{1}}+\frac{j_{2}}{P_{2}}$, with complete coion exclusion $\left(j_{2}=-j_{1}\right)$,

$j_{1}=S_{j} \frac{P_{1} P_{2}}{P_{2}-P_{1}}=S_{j} \frac{\sqrt{P_{1} P_{2}}}{\alpha-\frac{1}{\alpha}}$

Additionally,

$j_{1}=\frac{S_{j}}{C_{o}} * \frac{C_{o} \sqrt{P_{1} P_{2}}}{\alpha-\frac{1}{\alpha}}=C_{0} \frac{\sqrt{P_{1} P_{2}}}{\alpha-\frac{1}{\alpha}} \cdot\left(y_{0}+\beta y_{1}\right)$ 
Substituting for $y_{1}$ using $\mathrm{Eq}(\mathrm{S} 86)$ and the definition of $\beta$ (Eq(68) gives

$j_{1}=C_{0} \frac{\sqrt{P_{1} P_{2}}}{\alpha-\frac{1}{\alpha}} \cdot\left(y_{0}-\frac{L}{c_{X} / C_{0}} \cdot \sqrt{\frac{P_{1} P_{2}}{\bar{D}_{1} \bar{D}_{2}}}\left(\frac{2 \alpha^{2}}{\left(\alpha^{2}-1\right)(1+\alpha)^{2}}\left[\left(\bar{\alpha}-\frac{1}{\bar{\alpha}}\right)-\left(\frac{2}{1+\alpha}\right)^{2} \cdot\left(\bar{\alpha}-\frac{\alpha^{2}}{\bar{\alpha}}\right) \cdot\right] y_{0}\right)\right)$

Substituting $y_{0}=\left(\frac{\alpha-1}{\alpha+1}\right)$ (see Eq(S54)) and using the definitions of $\alpha, \bar{\alpha} \equiv \sqrt{\frac{P_{2}}{P_{1}}}, \sqrt{\frac{\bar{D}_{2}}{\bar{D}_{1}}}$, one obtains after extensive rearrangement

$j_{1}=\frac{C_{0}}{\left(\frac{1}{\sqrt{P_{1}}}+\frac{1}{\sqrt{P_{2}}}\right)^{2}}\left\{1-\frac{\frac{2 L}{\left(c_{X} / C_{0}\right)}}{\left(\sqrt{\frac{P_{2}}{P_{1}}}-\sqrt{\frac{P_{1}}{P_{2}}}\right)\left(\frac{1}{\sqrt{P_{1}}}+\frac{1}{\sqrt{P_{2}}}\right)^{2}} \cdot\left[\left(\frac{1}{\overline{\bar{D}_{1}}}-\frac{1}{\bar{D}_{2}}\right)-\left(\frac{2}{\sqrt{P_{1}}+\sqrt{P_{2}}}\right)^{2} \cdot\left(\frac{P_{1}}{\overline{\bar{D}_{1}}}-\frac{P_{2}}{\bar{D}_{2}}\right)\right]\right\}$

S9. Derivation of a first-order potential difference correction ( $\mathrm{Eq}(39))$ to the limiting case of constant electrochemical potentials: Bi-ionic potentials

This derivation begins with $\mathrm{Eq}(27)$, which we repeat below for convenience.

$\varphi(-1-L)-\varphi(1+L)=2\left[\frac{L \bar{S}_{j}}{c_{X}}+\ln \left(\frac{C_{0}+S_{j}}{C_{0}-S_{j}}\right)\right]$

We need to make small corrections to the values of $\bar{S}_{j}$ and $S_{j}$ obtained with the assumption of equal electrochemical potentials of counterions across the membrane. Based on Eq(S71) and taking only linear terms in $\beta$

$\frac{L \bar{S}_{j}}{c_{X}}=\beta y \cdot\left(\frac{\bar{\alpha}-\frac{1}{\bar{\alpha}}}{\alpha-\frac{1}{\alpha}}\right) \approx \beta y_{0} \cdot\left(\frac{\bar{\alpha}-\frac{1}{\bar{\alpha}}}{\alpha-\frac{1}{\alpha}}\right)$

For the last term in the brackets in $\mathrm{Eq}(27)$,

$\ln \left(\frac{C_{0}+S_{j}}{C_{0}-S_{j}}\right)=\ln \left(\frac{1+y}{1-y}\right) \approx \ln \left(\frac{1+y_{0}}{1-y_{0}}\right)+\ln \left(\frac{1+\frac{\beta y_{1}}{1+y_{0}}}{1-\frac{\beta y_{1}}{1-y_{0}}}\right) \approx \ln (\alpha)+\beta y_{1} \cdot\left(\frac{1}{1+y_{0}}+\frac{1}{1-y_{0}}\right)=\ln (\alpha)+2 \beta y_{1}$.

$\left(\frac{1}{1-y_{0}^{2}}\right)=\ln (\alpha)+\frac{\beta y_{1} \cdot(\alpha+1)^{2}}{2 \alpha}$

$\mathrm{Eq}(\mathrm{S} 92)$ uses the approximation that $\ln (1+x)=x$ and $\ln \left(\frac{1}{1-x}\right)=x$ for small $\mathrm{x}$. It also includes $y_{0}=$ $\frac{\alpha-1}{\alpha+1}$ so $\alpha=\frac{1+y_{0}}{1-y_{0}}$.

Substituting $\mathrm{Eq}(\mathrm{S} 92)$ and $\mathrm{Eq}(\mathrm{S} 91)$ into $\mathrm{Eq}(27)$ gives

$\varphi(-1-L)-\varphi(1+L) \approx 2\left[\beta y_{0} \cdot\left(\frac{\bar{\alpha}-\frac{1}{\bar{\alpha}}}{\alpha-\frac{1}{\alpha}}\right)+\ln (\alpha)+\frac{\beta y_{1} \cdot(\alpha+1)^{2}}{2 \alpha}\right]$

$\mathrm{Eq}(\mathrm{S} 86)$ gives an expression for $y_{1}$. Use of this expression in $\mathrm{Eq}(\mathrm{S} 93)$ leads to 
$\varphi(-1-L)-\varphi(1+L) \approx 2\left[\ln (\alpha)+\beta y_{0} \cdot\left\{\left(\frac{\bar{\alpha}-\frac{1}{\bar{\alpha}}}{\alpha-\frac{1}{\alpha}}\right)-\frac{\alpha}{\left(\alpha^{2}-1\right)}\left[\left(\bar{\alpha}-\frac{1}{\bar{\alpha}}\right)-\left(\frac{2}{\frac{1}{\sqrt{\alpha}}+\sqrt{\alpha}}\right)^{2} \cdot\left(\frac{\bar{\alpha}}{\alpha}-\frac{\alpha}{\bar{\alpha}}\right)\right]\right\}\right]$

Substituting $y_{0}=\frac{\alpha-1}{\alpha+1}$ and rearranging gives

$\varphi(-1-L)-\varphi(1+L) \approx 2\left[\ln (\alpha)+\beta \cdot \frac{1}{4}\left(\frac{2}{\frac{1}{\sqrt{\alpha}}+\sqrt{\alpha}}\right)^{4} \cdot\left(\frac{\bar{\alpha}}{\alpha}-\frac{\alpha}{\bar{\alpha}}\right)\right]$

Substituting $\beta \equiv \frac{L}{c_{X} / C_{0}} \cdot \sqrt{\frac{P_{1} P_{2}}{\bar{D}_{1} \bar{D}_{2}}}$ and $\alpha, \bar{\alpha} \equiv \sqrt{\frac{P_{2}}{P_{1}}}, \sqrt{\frac{\bar{D}_{2}}{\bar{D}_{1}}}$ and rearranging finally yields

$\varphi(-1-L)-\varphi(1+L)=\ln \left(\frac{P_{2}}{P_{1}}\right)+\frac{8 L C_{0}}{c_{X}} \cdot \frac{\frac{P_{1}}{\bar{D}_{1}}-\frac{P_{2}}{\bar{D}_{2}}}{\left(\sqrt[4]{\left.\frac{P_{2}}{P_{1}}+\sqrt[4]{\frac{P_{1}}{P_{2}}}\right)^{4}}\right.}$

S10. Derivation of $\operatorname{Eqs}(42,43)$ for the flux and potential drop during current passage in the limiting case of constant electrochemical potentials across an ion-exchange membrane

For a membrane flanked by boundary layers and two perfectly stirred reservoirs with the same solution composition,

$C_{\Delta, l}=C_{\Delta, r}=C_{\Delta, o}$

In this case, $\mathrm{Eq}(\mathrm{S} 53)$ becomes

$\left(C_{\Delta, o}-\frac{S_{\Delta} \cdot C_{0}}{2 S_{j}}\right) \cdot \frac{1}{\left(C_{0}-S_{j}\right)^{2}}=\left(C_{\Delta, o}-\frac{S_{\Delta} \cdot C_{0}}{2 S_{j}}\right) \cdot \frac{1}{\left(C_{0}+S_{j}\right)^{2}}$

Because $\frac{1}{\left(C_{0}-S_{j}\right)^{2}}$ will in general not equal $\frac{1}{\left(C_{0}+S_{j}\right)^{2}}$, then

$C_{\Delta, o}-\frac{s_{\Delta} \cdot C_{0}}{2 S_{j}}=0$ so $\frac{s_{\Delta}}{S_{j}}=\frac{2 C_{\Delta, o}}{C_{0}}$

Using Eq(40),,$j_{2}=\tilde{I}-j_{1}$, and the definitions of $S_{\Delta}, S_{j}$, and $C_{\Delta, o}$, and $C_{0}=2 c_{10}+2 c_{20}$, one can show that

$\frac{S_{\Delta}}{S_{j}} \equiv \frac{j_{1} \cdot\left(\frac{P_{2}}{P_{1}}+1\right)-\tilde{I}}{j_{1} \cdot\left(\frac{P_{2}}{P_{1}}-1\right)+\tilde{I}}=\frac{2 C_{\Delta, o}}{C_{0}}=\frac{c_{10}-c_{20}}{c_{10}+c_{20}}$

Accordingly, solving for $j_{1}$ gives

$j_{1}=\frac{\tilde{I}}{\frac{P_{2} \cdot c_{20}}{P_{1} \cdot c_{10}}+1}=\frac{\tilde{I} P_{1} \cdot c_{10}}{P_{2} \cdot c_{20}+P_{1} \cdot c_{10}}$

Using the definition of $S_{j}$, Eq(40), and Eq(S101), we obtain 
$S_{j} \equiv j_{1}\left(\frac{1}{P_{1}}-\frac{1}{P_{2}}\right)+\frac{\tilde{I}}{P_{2}}=\tilde{I}\left[\frac{P_{1} c_{10}}{P_{1} c_{10}+P_{2} c_{20}}\left(\frac{1}{P_{1}}-\frac{1}{P_{2}}\right)+\frac{1}{P_{2}}\right]=\frac{\tilde{I}}{P_{2}}\left[\frac{c_{10}\left(P_{2}-P_{1}\right)}{P_{1} c_{10}+P_{2} c_{20}}+1\right]=\frac{\tilde{I}}{P_{2}} \frac{c_{10}\left(P_{2}-P_{1}\right)+P_{1} c_{10}+P_{2} c_{20}}{P_{1} c_{10}+P_{2} c_{20}}=$ $\tilde{I} \frac{c_{10}+c_{20}}{P_{1} c_{10}+P_{2} c_{20}}=\frac{C_{0} \tilde{I} / 2}{P_{1} c_{10}+P_{2} c_{20}}$

Substituting Eq(S102) into $\mathrm{Eq}(21), \varphi(-1)-\varphi(+1)=2 \ln \left(\frac{C_{0}+S_{j}}{C_{0}-S_{j}}\right)$, yields

$\varphi(-1)-\varphi(1)=2 \ln \left(\frac{C_{0}+S_{j}}{C_{0}-S_{j}}\right)=2 \ln \left(\frac{C_{0}+\frac{C_{0} \tilde{I} / 2}{P_{1} c_{10}+P_{2} c_{20}}}{C_{0}-\frac{C_{0} \tilde{I} / 2}{P_{1} c_{10}+P_{2} c_{20}}}\right)=2 \ln \left(\frac{1+\frac{\tilde{I} / 2}{P_{1} c_{10}+P_{2} c_{20}}}{1-\frac{\tilde{I} / 2}{P_{1} c_{10}+P_{2} c_{20}}}\right)=2 \ln \left(\frac{P_{1} c_{10}+P_{2} c_{20}+\tilde{I} / 2}{P_{1} c_{10}+P_{2} c_{20}-\tilde{I} / 2}\right)$

S11. Derivation of Eq(45) for the flux of ion "1" during current passage: First-order correction to the limiting case of constant electrochemical potentials across an ion-exchange membrane

As with the bi-ionic potential, this derivation starts with $\mathrm{Eq}(\mathrm{S} 40)$, which we repeat hear for convenience.

$\left(\frac{C_{\Delta, l}}{C_{0}}-\frac{S_{\Delta}}{2 S_{j}}\right) \cdot\left(\frac{C_{0}}{C_{0}-S_{j}}\right)^{2} \cdot \exp \left(\frac{L \bar{S}_{j}}{c_{X}}\right)-\left(\frac{c_{\Delta, r}}{C_{0}}-\frac{S_{\Delta}}{2 S_{j}}\right) \cdot\left(\frac{C_{0}}{C_{0}+S_{j}}\right)^{2} \cdot \exp \left(-\frac{L \bar{S}_{j}}{c_{X}}\right)=-\left(\frac{s_{\Delta}}{S_{j}}-\frac{\bar{S}_{\Delta}}{\bar{S}_{j}}\right) \cdot \sinh \left(\frac{L \bar{S}_{j}}{c_{X}}\right)$

We again assume that the term $\frac{L \bar{S}_{j}}{c_{X}}$ is small, which leads to the linear approximations described previously.

$\sinh \left(\frac{L \bar{S}_{j}}{c_{X}}\right)=\frac{L \bar{S}_{j}}{c_{X}} ; \exp \left(\frac{L \bar{S}_{j}}{c_{X}}\right)=1+\frac{L \bar{S}_{j}}{c_{X}} ; \exp \left(-\frac{L \bar{S}_{j}}{c_{X}}\right)=1-\frac{L \bar{S}_{j}}{c_{X}}$

Substituting these approximations gives

$\left(\frac{C_{\Delta, l}}{C_{0}}-\frac{S_{\Delta}}{2 S_{j}}\right) \cdot\left(\frac{C_{0}}{C_{0}-S_{j}}\right)^{2}+\frac{L \bar{S}_{j}}{c_{X}}\left(\frac{C_{\Delta, l}}{C_{0}}-\frac{S_{\Delta}}{2 S_{j}}\right) \cdot\left(\frac{C_{0}}{C_{0}-S_{j}}\right)^{2}-\left(\frac{C_{\Delta, r}}{C_{0}}-\frac{S_{\Delta}}{S_{j}}\right) \cdot\left(\frac{C_{0}}{C_{0}+S_{j}}\right)^{2}+\frac{L \bar{S}_{j}}{c_{X}}\left(\frac{C_{\Delta, r}}{C_{0}}-\frac{S_{\Delta}}{S_{j}}\right) \cdot\left(\frac{C_{0}}{C_{0}+S_{j}}\right)^{2}+$ $\left(\frac{s_{\Delta}}{s_{j}}-\frac{\bar{s}_{\Delta}}{\bar{S}_{j}}\right) \cdot \frac{L \bar{S}_{j}}{c_{X}}=0$

Using $C_{\Delta, l}=C_{\Delta r}=C_{\Delta, o}, \mathrm{Eq}(\mathrm{S} 97)$, and rearranging gives

$\left(\frac{C_{\Delta, o}}{C_{0}}-\frac{S_{\Delta}}{2 S_{j}}\right)\left[\left(\frac{C_{0}}{C_{0}-S_{j}}\right)^{2}-\left(\frac{C_{0}}{C_{0}+S_{j}}\right)^{2}\right]+\frac{L \bar{S}_{j}}{c_{X}}\left\{\left(\frac{C_{\Delta, o}}{C_{0}}-\frac{S_{\Delta}}{2 S_{j}}\right) \cdot\left[\left(\frac{C_{0}}{C_{0}-S_{j}}\right)^{2}+\left(\frac{C_{0}}{C_{0}+S_{j}}\right)^{2}\right]+\left(\frac{S_{\Delta}}{S_{j}}-\frac{\bar{s}_{\Delta}}{\bar{S}_{j}}\right)\right\}=0$

We will look at the different terms in this expression to eventually solve for the fluxes of individual ions. First, we remember that for applications of a constant current

$j_{2}=\tilde{I}-j_{1}$

Accordingly based on the definitions of $S_{j}$ and $\bar{S}_{j}$ (sums of fluxes divided by permeances)

$$
\begin{aligned}
& S_{j} \equiv j_{1} \cdot\left(\frac{1}{P_{1}}-\frac{1}{P_{2}}\right)+\frac{\tilde{I}}{P_{2}} \\
& \bar{S}_{j} \equiv j_{1} \cdot\left(\frac{1}{\bar{D}_{1}}-\frac{1}{\bar{D}_{2}}\right)+\frac{\tilde{I}}{\bar{D}_{2}}
\end{aligned}
$$


$j_{1} \equiv \frac{S_{j}-\frac{\tilde{I}}{P_{2}}}{\left(\frac{1}{P_{1}}-\frac{1}{P_{2}}\right)}$

The use of $\mathrm{Eq}(\mathrm{S} 108)$ in $\mathrm{Eq}(\mathrm{S} 107)$ gives

$\bar{S}_{j} \equiv \frac{S_{j}-\frac{\tilde{I}}{P_{2}}}{\left(\frac{1}{P_{1}}-\frac{1}{P_{2}}\right)} \cdot\left(\frac{1}{\bar{D}_{1}}-\frac{1}{\bar{D}_{2}}\right)+\frac{\tilde{I}}{\bar{D}_{2}}$

With sufficient identical transformations and substitution of $\alpha, \bar{\alpha} \equiv \sqrt{\frac{P_{2}}{P_{1}}}, \sqrt{\frac{\bar{D}_{2}}{\bar{D}_{1}}}$ into this equation, we obtain

$\bar{S}_{j} \equiv \sqrt{\frac{P_{1} P_{2}}{\bar{D}_{1} \bar{D}_{2}}} \cdot\left[S_{j} \cdot\left(\frac{\bar{\alpha}-\frac{1}{\bar{\alpha}}}{\alpha-\frac{1}{\alpha}}\right)+\frac{\tilde{I}}{\sqrt{P_{1} P_{2}}} \cdot \frac{\frac{\alpha}{\bar{\alpha}}-\frac{\bar{\alpha}}{\alpha}}{\alpha-\frac{1}{\alpha}}\right]$

As in prior derivations of corrections to bionic potentials, defining $y \equiv S_{j} / C_{0}$ leads to

$\frac{\bar{S}_{j}}{C_{0}} \equiv \sqrt{\frac{P_{1} P_{2}}{\bar{D}_{1} \bar{D}_{2}}} \cdot\left[y \cdot\left(\frac{\bar{\alpha}-\frac{1}{\bar{\alpha}}}{\alpha-\frac{1}{\alpha}}\right)+\check{I} \cdot\left(\frac{\frac{\alpha}{\bar{\alpha}}-\frac{\bar{\alpha}}{\alpha}}{\alpha-\frac{1}{\alpha}}\right)\right]$

where we define a dimensionless current, $\check{I}$

$\check{I}=\frac{\tilde{I}}{C_{0} \sqrt{P_{1} P_{2}}}$

Using $\mathrm{Eq}(\mathrm{S} 111)$ and the definition $\beta \equiv \frac{L}{c_{X} / C_{0}} \cdot \sqrt{\frac{P_{1} P_{2}}{\bar{D}_{1} \bar{D}_{2}}}$

$\frac{L \bar{S}_{j}}{c_{X}}=L \frac{\bar{S}_{j} / C_{0}}{c_{X} / C_{0}} \equiv \frac{L}{c_{X} / C_{0}} \sqrt{\frac{P_{1} P_{2}}{\bar{D}_{1} \bar{D}_{2}}} \cdot\left[y \cdot\left(\frac{\bar{\alpha}-\frac{1}{\bar{\alpha}}}{\alpha-\frac{1}{\alpha}}\right)+\check{I} \cdot\left(\frac{\frac{\alpha}{\bar{\alpha}}-\frac{\bar{\alpha}}{\alpha}}{\alpha-\frac{1}{\alpha}}\right)\right]=\beta \cdot\left[y \cdot\left(\frac{\bar{\alpha}-\frac{1}{\bar{\alpha}}}{\alpha-\frac{1}{\alpha}}\right)+\check{I} \cdot\left(\frac{\frac{\alpha}{\bar{\alpha}}-\frac{\bar{\alpha}}{\alpha}}{\alpha-\frac{1}{\alpha}}\right)\right]$

Substituting $\mathrm{Eq}(\mathrm{S} 108)$ and $\mathrm{Eq}(40)$ into the definition of $S_{\Delta}$

$S_{\Delta}=\frac{S_{j}-\frac{\tilde{I}}{P_{2}}}{\left(\frac{1}{P_{1}}-\frac{1}{P_{2}}\right)} \cdot\left(\frac{1}{P_{1}}+\frac{1}{P_{2}}\right)-\frac{\tilde{I}}{P_{2}}$

With appropriate transformations and substitution of $\alpha=\sqrt{\frac{P_{2}}{P_{1}}}$,

$S_{\Delta}=S_{j} \cdot \frac{\frac{1}{P_{1}}+\frac{1}{P_{2}}}{\frac{1}{P_{1}}-\frac{1}{P_{2}}}-\frac{\tilde{I}}{P_{2}} \cdot\left(1+\frac{\frac{1}{P_{1}}+\frac{1}{P_{2}}}{\frac{1}{P_{1}}-\frac{1}{P_{2}}}\right)=S_{j} \cdot \frac{P_{2}+P_{1}}{P_{2}-P_{1}}-2 \tilde{I} \cdot\left(\frac{1}{P_{2}-P_{1}}\right)=S_{j} \cdot \frac{\alpha+\frac{1}{\alpha}}{\alpha-\frac{1}{\alpha}}-\frac{2 \tilde{I}}{\sqrt{P_{1} P_{2}}} \cdot\left(\frac{1}{\alpha-\frac{1}{\alpha}}\right)$

Finally, dividing by both sides by $S_{j}$ and substituting the definition of $y \equiv S_{j} / C_{0}$ and $\check{I}=\frac{\tilde{I}}{C_{0} \sqrt{P_{1} P_{2}}}$ yields

$\frac{S_{\Delta}}{S_{j}} \equiv\left(\frac{\alpha+\frac{1}{\alpha}}{\alpha-\frac{1}{\alpha}}\right)-\frac{1}{y} \cdot\left(\frac{2 \check{I}}{\alpha-\frac{1}{\alpha}}\right)$ 
Using a similar procedure

$\bar{S}_{\Delta} \equiv \bar{S}_{j} \cdot\left(\frac{\bar{\alpha}+\frac{1}{\bar{\alpha}}}{\bar{\alpha}-\frac{1}{\bar{\alpha}}}\right)-\frac{2 \tilde{I}}{\sqrt{\bar{D}_{1} \bar{D}_{2}}} \cdot\left(\frac{1}{\bar{\alpha}-\frac{1}{\bar{\alpha}}}\right)$

Dividing both sized of $\mathrm{Eq}(117)$ by $\bar{S}_{j}$ and in the second term on the right substituting for $\bar{S}_{j}$ from $\mathrm{Eq}(\mathrm{S} 113)$ leads to

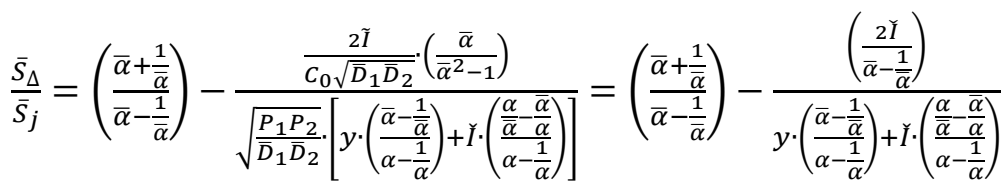

Looking at $\mathrm{Eq}(\mathrm{S} 105)$, which we repeat here for convenience,

$\left(\frac{C_{\Delta, o}}{C_{0}}-\frac{S_{\Delta}}{2 S_{j}}\right)\left[\left(\frac{C_{0}}{C_{0}-S_{j}}\right)^{2}-\left(\frac{C_{0}}{C_{0}+S_{j}}\right)^{2}\right]+\frac{L \bar{S}_{j}}{C_{X}}\left\{\left(\frac{C_{\Delta, o}}{C_{0}}-\frac{S_{\Delta}}{2 S_{j}}\right) \cdot\left[\left(\frac{C_{0}}{C_{0}-S_{j}}\right)^{2}+\left(\frac{C_{0}}{C_{0}+S_{j}}\right)^{2}\right]+\left(\frac{S_{\Delta}}{S_{j}}-\frac{\bar{S}_{\Delta}}{\bar{S}_{j}}\right)\right\}=0$

Considering the limiting case where $\frac{L}{c_{X}}$ approaches zero, $\left(\frac{C_{\Delta, o}}{C_{0}}-\frac{s_{\Delta}}{S_{j}}\right)$ must be zero so the first term goes to zero. Thus, for small linear corrections, $\left(\frac{C_{\Delta, o}}{C_{0}}-\frac{s_{\Delta}}{S_{j}}\right)$ should be small. In this approximation

$\frac{L \bar{S}_{j}}{c_{X}}\left(\frac{C_{\Delta, o}}{C_{0}}-\frac{S_{\Delta}}{2 S_{j}}\right) \cdot\left[\left(\frac{C_{0}}{C_{0}-S_{j}}\right)^{2}+\left(\frac{C_{0}}{C_{0}+S_{j}}\right)^{2}\right] \approx 0$

because it contains the product of two small terms, namely $\frac{L \bar{S}_{j}}{c_{X}}$ and $\left(\frac{C_{\Delta, o}}{C_{0}}-\frac{S_{\Delta}}{2 S_{j}}\right)$. Taking into account $\mathrm{Eq}(\mathrm{S} 119), \mathrm{Eq}(\mathrm{S} 105)$ becomes

$\left(\frac{C_{\Delta, o}}{C_{0}}-\frac{S_{\Delta}}{2 S_{j}}\right)\left[\left(\frac{C_{0}}{C_{0}-S_{j}}\right)^{2}-\left(\frac{C_{0}}{C_{0}+S_{j}}\right)^{2}\right]+\frac{L \bar{S}_{j}}{c_{X}}\left(\frac{S_{\Delta}}{S_{j}}-\frac{\bar{S}_{\Delta}}{\bar{S}_{j}}\right) \approx 0$

With the substitution that $y=S_{j} / C_{0}$,

$\left(\frac{C_{0}}{C_{0}-S_{j}}\right)^{2}-\left(\frac{C_{0}}{C_{0}+S_{j}}\right)^{2}=\left(\frac{1}{1-y}\right)^{2}-\left(\frac{1}{1+y}\right)^{2}=\frac{4 y}{\left(1-y^{2}\right)^{2}}$

Substituting $\mathrm{Eq}(121)$ and $\mathrm{Eq}(\mathrm{S} 113)$ into $\mathrm{Eq}(\mathrm{S} 120)$ gives

$\left(2 \frac{C_{\Delta, o}}{C_{0}}-\frac{s_{\Delta}}{S_{j}}\right) \cdot \frac{4 y}{\left(1-y^{2}\right)^{2}}+2\left(\frac{s_{\Delta}}{s_{j}}-\frac{\bar{s}_{\Delta}}{\bar{S}_{j}}\right) \cdot \beta \cdot\left[y \cdot\left(\frac{\bar{\alpha}-\frac{1}{\bar{\alpha}}}{\alpha-\frac{1}{\alpha}}\right)+\check{I} \cdot\left(\frac{\frac{\alpha}{\bar{\alpha}}-\frac{\bar{\alpha}}{\alpha}}{\alpha-\frac{1}{\alpha}}\right)\right] \approx 0$

Identical transformations lead to 


$$
\begin{aligned}
& 2\left(\frac{S_{\Delta}}{S_{j}}-\frac{\bar{S}_{\Delta}}{\bar{S}_{j}}\right) \cdot\left[y \cdot\left(\frac{\bar{\alpha}-\frac{1}{\bar{\alpha}}}{\alpha-\frac{1}{\alpha}}\right)+\check{I} \cdot\left(\frac{\frac{\alpha}{\bar{\alpha}}-\frac{\bar{\alpha}}{\alpha}}{\alpha-\frac{1}{\alpha}}\right)\right]=2\left\{\left(\frac{\alpha+\frac{1}{\alpha}}{\alpha-\frac{1}{\alpha}}\right)-\left(\frac{\bar{\alpha}+\frac{1}{\bar{\alpha}}}{\bar{\alpha}-\frac{1}{\bar{\alpha}}}\right)+\frac{\left(\frac{2 \tilde{I}}{\bar{\alpha}-\frac{1}{\bar{\alpha}}}\right)}{y \cdot\left(\frac{\bar{\alpha}-\frac{1}{\alpha}}{\alpha-\frac{1}{\alpha}}\right)+\tilde{I} \cdot\left(\frac{\frac{\alpha}{\alpha}-\frac{\bar{\alpha}}{\alpha}}{\alpha-\frac{1}{\alpha}}\right)}-\frac{1}{y} \cdot\left(\frac{2 \check{I}}{\alpha-\frac{1}{\alpha}}\right)\right\}\left[y \cdot\left(\frac{\bar{\alpha}-\frac{1}{\bar{\alpha}}}{\alpha-\frac{1}{\alpha}}\right)+\right. \\
& \left.\check{I} \cdot\left(\frac{\frac{\alpha}{\bar{\alpha}}-\frac{\bar{\alpha}}{\alpha}}{\alpha-\frac{1}{\alpha}}\right)\right]=2\left[\left(\frac{\alpha+\frac{1}{\alpha}}{\alpha-\frac{1}{\alpha}}\right)-\left(\frac{\bar{\alpha}+\frac{1}{\bar{\alpha}}}{\bar{\alpha}-\frac{1}{\bar{\alpha}}}\right)-\frac{1}{y} \cdot\left(\frac{2 \check{I}}{\alpha-\frac{1}{\alpha}}\right)\right] \cdot\left[y \cdot\left(\frac{\bar{\alpha}-\frac{1}{\bar{\alpha}}}{\alpha-\frac{1}{\alpha}}\right)+\check{I} \cdot\left(\frac{\frac{\alpha}{\bar{\alpha}}-\frac{\bar{\alpha}}{\alpha}}{\alpha-\frac{1}{\alpha}}\right)\right]+\frac{4 \check{I}}{\bar{\alpha}-\frac{1}{\bar{\alpha}}}
\end{aligned}
$$

Noting that $\left(\frac{\alpha+\frac{1}{\alpha}}{\alpha-\frac{1}{\alpha}}\right)-\left(\frac{\bar{\alpha}+\frac{1}{\bar{\alpha}}}{\bar{\alpha}-\frac{1}{\bar{\alpha}}}\right) \equiv \frac{\alpha \bar{\alpha}-\frac{\alpha}{\bar{\alpha}}+\frac{\bar{\alpha}}{\alpha}-\frac{1}{\alpha \bar{\alpha}}-\left(\alpha \bar{\alpha}+\frac{\alpha}{\bar{\alpha}}-\frac{\bar{\alpha}}{\alpha}-\frac{1}{\alpha \bar{\alpha}}\right)}{\left(\alpha-\frac{1}{\alpha}\right)\left(\bar{\alpha}-\frac{1}{\bar{\alpha}}\right)} \equiv-\frac{2\left(\frac{\alpha}{\bar{\alpha}}-\frac{\bar{\alpha}}{\alpha}\right)}{\left(\alpha-\frac{1}{\alpha}\right)\left(\bar{\alpha}-\frac{1}{\bar{\alpha}}\right)^{\prime}}$

$2\left(\frac{s_{\Delta}}{s_{j}}-\frac{\bar{S}_{\Delta}}{\bar{S}_{j}}\right) \cdot\left[y \cdot\left(\frac{\bar{\alpha}-\frac{1}{\bar{\alpha}}}{\alpha-\frac{1}{\alpha}}\right)+\check{I} \cdot\left(\frac{\frac{\alpha}{\bar{\alpha}}-\frac{\bar{\alpha}}{\alpha}}{\alpha-\frac{1}{\alpha}}\right)\right]=2\left[\left(\frac{\alpha+\frac{1}{\alpha}}{\alpha-\frac{1}{\alpha}}\right)-\left(\frac{\bar{\alpha}+\frac{1}{\bar{\alpha}}}{\bar{\alpha}-\frac{1}{\bar{\alpha}}}\right)-\frac{1}{y} \cdot\left(\frac{2 \check{I}}{\alpha-\frac{1}{\alpha}}\right)\right] \cdot\left[y \cdot\left(\frac{\bar{\alpha}-\frac{1}{\bar{\alpha}}}{\alpha-\frac{1}{\alpha}}\right)+\check{I} \cdot\left(\frac{\frac{\alpha}{\bar{\alpha}}-\frac{\bar{\alpha}}{\alpha}}{\alpha-\frac{1}{\alpha}}\right)\right]+$

$\frac{4 \check{I}}{\bar{\alpha}-\frac{1}{\bar{\alpha}}}=2\left[-\frac{2\left(\frac{\alpha}{\bar{\alpha}}-\frac{\bar{\alpha}}{\alpha}\right)}{\left(\alpha-\frac{1}{\alpha}\right)\left(\bar{\alpha}-\frac{1}{\bar{\alpha}}\right)}-\frac{1}{y} \cdot\left(\frac{2 \check{I}}{\alpha-\frac{1}{\alpha}}\right)\right] \cdot\left[y \cdot\left(\frac{\bar{\alpha}-\frac{1}{\bar{\alpha}}}{\alpha-\frac{1}{\alpha}}\right)+\tilde{I} \cdot\left(\frac{\frac{\alpha}{\bar{\alpha}}-\frac{\bar{\alpha}}{\alpha}}{\alpha-\frac{1}{\alpha}}\right)\right]+\frac{4 \check{I}}{\bar{\alpha}-\frac{1}{\bar{\alpha}}}=-\frac{4}{\left(\alpha-\frac{1}{\alpha}\right)^{2}}\left[\frac{\left(\frac{\alpha}{\bar{\alpha}}-\frac{\bar{\alpha}}{\alpha}\right)}{\left(\bar{\alpha}-\frac{1}{\bar{\alpha}}\right)}+\left(\frac{\check{I}}{y}\right)\right]$.

$\left[y \cdot\left(\bar{\alpha}-\frac{1}{\bar{\alpha}}\right)+\check{I} \cdot\left(\frac{\alpha}{\bar{\alpha}}-\frac{\bar{\alpha}}{\alpha}\right)\right]+\frac{4 \check{I}}{\bar{\alpha}-\frac{1}{\bar{\alpha}}}=-\frac{4}{\left(\alpha-\frac{1}{\alpha}\right)^{2}}\left[y \cdot\left(\frac{\alpha}{\bar{\alpha}}-\frac{\bar{\alpha}}{\alpha}\right)+\check{I} \cdot \frac{\left(\frac{\alpha}{\bar{\alpha}}-\frac{\bar{\alpha}}{\alpha}\right)^{2}}{\left(\bar{\alpha}-\frac{1}{\bar{\alpha}}\right)}+\check{I} \cdot\left(\bar{\alpha}-\frac{1}{\bar{\alpha}}\right)+\frac{\check{I}^{2}}{y} \cdot\left(\frac{\alpha}{\bar{\alpha}}-\frac{\bar{\alpha}}{\alpha}\right)\right]+\frac{4 \check{I}}{\bar{\alpha}-\frac{1}{\bar{\alpha}}}$

In the absence of small corrections, i.e. in the limiting case where $L$ approaches zero, the first term of $\mathrm{Eq}(105),\left(\frac{C_{\Delta, o}}{C_{0}}-\frac{S_{\Delta}}{2 S_{j}}\right)$ must be zero. Using Eq(116)

$\frac{2 C_{\Delta, o}}{C_{0}}=\frac{S_{\Delta}}{S_{j}}=\left(\frac{\alpha+\frac{1}{\alpha}}{\alpha-\frac{1}{\alpha}}\right)-\frac{1}{y_{o}} \cdot\left(\frac{2 \check{I}}{\alpha-\frac{1}{\alpha}}\right)$

Solving $\mathrm{Eq}(\mathrm{S} 125)$ for $y_{0}$ yields

$y_{0}=\frac{2 \alpha \check{I}}{\left(\alpha^{2}+1\right)-2 \frac{C_{\Delta, o}}{C_{0}}\left(\alpha^{2}-1\right)}=\alpha A \check{I}$

$A=\frac{2}{\left(\alpha^{2}+1\right)-2 \frac{C_{\Delta, o}}{C_{0}}\left(\alpha^{2}-1\right)}=\frac{1}{f+\alpha^{2} \cdot(1-f)} ; f=\frac{c_{10}}{c_{10}+c_{20}}$

In Eq(S122), we multiply $2\left(\frac{s_{\Delta}}{s_{j}}-\frac{\bar{s}_{\Delta}}{\bar{S}_{j}}\right) \cdot\left[y \cdot\left(\frac{\bar{\alpha}-\frac{1}{\bar{\alpha}}}{\alpha-\frac{1}{\alpha}}\right)+\check{I} \cdot\left(\frac{\frac{\alpha}{\bar{\alpha}}-\frac{\bar{\alpha}}{\alpha}}{\alpha-\frac{1}{\alpha}}\right)\right]$ by $\beta$, so we can approximate $y_{0}$ for $y$ in Eq(S124). This leads to

$2\left(\frac{s_{\Delta}}{s_{j}}-\frac{\bar{S}_{\Delta}}{\bar{S}_{j}}\right) \cdot\left[y \cdot\left(\frac{\bar{\alpha}-\frac{1}{\bar{\alpha}}}{\alpha-\frac{1}{\alpha}}\right)+\check{I} \cdot\left(\frac{\frac{\alpha}{\bar{\alpha}}-\frac{\bar{\alpha}}{\alpha}}{\alpha-\frac{1}{\alpha}}\right)\right]=-\frac{4 \check{I}}{\left(\alpha-\frac{1}{\alpha}\right)^{2}}\left[\alpha A \cdot\left(\frac{\alpha}{\bar{\alpha}}-\frac{\bar{\alpha}}{\alpha}\right)+\frac{\left(\frac{\alpha}{\bar{\alpha}}-\frac{\bar{\alpha}}{\alpha}\right)^{2}}{\left(\bar{\alpha}-\frac{1}{\bar{\alpha}}\right)}+\left(\bar{\alpha}-\frac{1}{\bar{\alpha}}\right)+\frac{1}{\alpha A} \cdot\left(\frac{\alpha}{\bar{\alpha}}-\frac{\bar{\alpha}}{\alpha}\right)\right]+\frac{4 \check{I}}{\bar{\alpha}-\frac{1}{\bar{\alpha}}}$

In Eq(S122), with the correction, the term $\left(\frac{C_{\Delta, o}}{C_{0}}-\frac{s_{\Delta}}{S_{j}}\right)$ will be small. Thus, we can also susbstitute $y_{0}$ for $y$ in the term $\frac{4 y}{\left(1-y^{2}\right)^{2}}$. Finally, we return to $\frac{S_{\Delta}}{S_{j}} \equiv\left(\frac{\alpha+\frac{1}{\alpha}}{\alpha-\frac{1}{\alpha}}\right)-\frac{1}{y} \cdot\left(\frac{2 \check{I}}{\alpha-\frac{1}{\alpha}}\right)$ in Eq(S116). With the substitution of $y=y_{0}+\beta y_{1}$, the expression becomes 
$\frac{s_{\Delta}}{s_{j}} \equiv\left(\frac{\alpha+\frac{1}{\alpha}}{\alpha-\frac{1}{\alpha}}\right)-\frac{1}{y_{0}+\beta y_{1}} \cdot\left(\frac{2 \check{I}}{\alpha-\frac{1}{\alpha}}\right)$

Using the approximation that for small $x, \frac{1}{1+x}=1-x$,

$\frac{S_{\Delta}}{S_{j}} \equiv\left(\frac{\alpha+\frac{1}{\alpha}}{\alpha-\frac{1}{\alpha}}\right)-\frac{1}{y_{0}\left(1+\frac{\beta y_{1}}{y_{0}}\right)} \cdot\left(\frac{2 \check{I}}{\alpha-\frac{1}{\alpha}}\right) \approx\left(\frac{\alpha+\frac{1}{\alpha}}{\alpha-\frac{1}{\alpha}}\right)-\left(\frac{2 \check{I}}{\alpha-\frac{1}{\alpha}}\right) \cdot \frac{1}{y_{0}}\left(1-\frac{\beta y_{1}}{y_{0}}\right)=\left[\left(\frac{\alpha+\frac{1}{\alpha}}{\alpha-\frac{1}{\alpha}}\right)-\left(\frac{2 \check{I}}{\alpha-\frac{1}{\alpha}}\right) \cdot \frac{1}{y_{0}}\right]+\left(\frac{2 \check{I}}{\alpha-\frac{1}{\alpha}}\right) \cdot \frac{\beta y_{1}}{y_{0}^{2}}$

Substituting from Eq(S125)

$\frac{S_{\Delta}}{S_{j}}=\frac{2 C_{\Delta, o}}{C_{0}}+\left(\frac{2 \check{I}}{\alpha-\frac{1}{\alpha}}\right) \cdot \frac{\beta y_{1}}{y_{0}^{2}}$

Thus,

$\left(\frac{2 C_{\Delta, o}}{C_{0}}-\frac{S_{\Delta}}{S_{j}}\right) \cdot \frac{4 y_{0}}{\left(1-y_{0}^{2}\right)^{2}} \approx-\frac{4 y_{0}}{\left(1-y_{0}^{2}\right)^{2}}\left(\frac{2 \check{I}}{\alpha-\frac{1}{\alpha}}\right) \cdot \frac{\beta y_{1}}{y_{0}^{2}}=-\left(\frac{8 \check{I}}{\alpha-\frac{1}{\alpha}}\right) \cdot \frac{\beta y_{1}}{y_{0} \cdot\left(1-y_{0}^{2}\right)^{2}}$

Substituting $\mathrm{Eq}(\mathrm{S} 132)$ and $\mathrm{Eq}(\mathrm{S} 128)$ int $\mathrm{Eq}(\mathrm{S} 122)$ and dividing both sided by $\beta$ leads to

$-\left(\frac{8 \check{I}}{\alpha-\frac{1}{\alpha}}\right) \cdot \frac{y_{1}}{y_{0}\left(1-y_{0}^{2}\right)^{2}}-\frac{4 \check{I}}{\left(\alpha-\frac{1}{\alpha}\right)^{2}}\left[\alpha A \cdot\left(\frac{\alpha}{\bar{\alpha}}-\frac{\bar{\alpha}}{\alpha}\right)+\frac{\left(\frac{\alpha}{\bar{\alpha}}-\frac{\bar{\alpha}}{\alpha}\right)^{2}}{\left(\bar{\alpha}-\frac{1}{\bar{\alpha}}\right)}+\left(\bar{\alpha}-\frac{1}{\bar{\alpha}}\right)+\frac{1}{\alpha A} \cdot\left(\frac{\alpha}{\bar{\alpha}}-\frac{\bar{\alpha}}{\alpha}\right)\right]+\frac{4 \check{I}}{\bar{\alpha}-\frac{1}{\bar{\alpha}}}=0$

Substituting $y_{0}=\frac{2 \alpha \check{I}}{\left(\alpha^{2}+1\right)-2 \frac{C_{\Delta, o}}{C_{0}}\left(\alpha^{2}-1\right)}=\alpha A \check{I}$ and multiplying by by $\left(\alpha-\frac{1}{\alpha}\right) / 4$ gives

$-\frac{2 y_{1}}{\alpha A \cdot\left(1-(\alpha A \check{I})^{2}\right)^{2}}-\frac{\check{I}}{\left(\alpha-\frac{1}{\alpha}\right)}\left[\alpha A \cdot\left(\frac{\alpha}{\bar{\alpha}}-\frac{\bar{\alpha}}{\alpha}\right)+\frac{\left(\frac{\alpha}{\bar{\alpha}}-\frac{\bar{\alpha}}{\alpha}\right)^{2}}{\left(\bar{\alpha}-\frac{1}{\bar{\alpha}}\right)}+\left(\bar{\alpha}-\frac{1}{\bar{\alpha}}\right)+\frac{1}{\alpha A} \cdot\left(\frac{\alpha}{\bar{\alpha}}-\frac{\bar{\alpha}}{\alpha}\right)\right]+\check{I} \cdot \frac{\left(\alpha-\frac{1}{\alpha}\right)}{\left(\bar{\alpha}-\frac{1}{\bar{\alpha}}\right)}=0($

Rearranging yields

$-\frac{2 y_{1}}{\alpha A\left(1-(\alpha A \check{I})^{2}\right)^{2}}-\frac{\check{I}}{\left(\alpha-\frac{1}{\alpha}\right)}\left[\left(\alpha A+\frac{1}{\alpha A}\right) \cdot\left(\frac{\alpha}{\bar{\alpha}}-\frac{\bar{\alpha}}{\alpha}\right)+\frac{\left(\frac{\alpha}{\bar{\alpha}}-\frac{\bar{\alpha}}{\alpha}\right)^{2}}{\left(\bar{\alpha}-\frac{1}{\bar{\alpha}}\right)}+\left(\bar{\alpha}-\frac{1}{\bar{\alpha}}\right)\right]+\check{I} \cdot \frac{\left(\alpha-\frac{1}{\alpha}\right)}{\left(\bar{\alpha}-\frac{1}{\bar{\alpha}}\right)}=0$

Through the series of transformations shown in the non-numbered equations below

$$
\begin{aligned}
& \left(\alpha A+\frac{1}{\alpha A}\right) \cdot\left(\frac{\alpha}{\bar{\alpha}}-\frac{\bar{\alpha}}{\alpha}\right)+\frac{\left(\frac{\alpha}{\bar{\alpha}}-\frac{\bar{\alpha}}{\alpha}\right)^{2}}{\left(\bar{\alpha}-\frac{1}{\bar{\alpha}}\right)} \equiv\left(\frac{\alpha}{\bar{\alpha}}-\frac{\bar{\alpha}}{\alpha}\right)\left[\left(\alpha A+\frac{1}{\alpha A}\right)+\frac{\frac{\alpha}{\bar{\alpha}}-\frac{\bar{\alpha}}{\alpha}}{\bar{\alpha}-\frac{1}{\bar{\alpha}}}\right] \equiv\left(\frac{\frac{\alpha}{\bar{\alpha}}-\frac{\bar{\alpha}}{\alpha}}{\bar{\alpha}-\frac{1}{\bar{\alpha}}}\right)\left[\left(\alpha A+\frac{1}{\alpha A}\right)\left(\bar{\alpha}-\frac{1}{\bar{\alpha}}\right)+\left(\frac{\alpha}{\bar{\alpha}}-\frac{\bar{\alpha}}{\alpha}\right)\right] \\
& -\frac{2 y_{1}}{\alpha A\left(1-(\alpha A \tilde{I})^{2}\right)^{2}}-\frac{\check{I}}{\left(\alpha-\frac{1}{\alpha}\right)}\left\{\left(\frac{\frac{\alpha}{\bar{\alpha}}-\frac{\bar{\alpha}}{\alpha}}{\bar{\alpha}-\frac{1}{\bar{\alpha}}}\right)\left[\left(\alpha A+\frac{1}{\alpha A}\right)\left(\bar{\alpha}-\frac{1}{\bar{\alpha}}\right)+\left(\frac{\alpha}{\bar{\alpha}}-\frac{\bar{\alpha}}{\alpha}\right)\right]+\left(\bar{\alpha}-\frac{1}{\bar{\alpha}}\right)\right\}+\check{I} \cdot \frac{\left(\alpha-\frac{1}{\alpha}\right)}{\left(\bar{\alpha}-\frac{1}{\bar{\alpha}}\right)}=0
\end{aligned}
$$




$$
\begin{aligned}
& -\frac{2 y_{1}}{\alpha A\left(1-(\alpha A \tilde{I})^{2}\right)^{2}}-\frac{\check{I}}{\left(\alpha-\frac{1}{\alpha}\right)\left(\bar{\alpha}-\frac{1}{\bar{\alpha}}\right)}\left\{\left(\frac{\alpha}{\bar{\alpha}}-\frac{\bar{\alpha}}{\alpha}\right)\left[\left(\alpha A+\frac{1}{\alpha A}\right)\left(\bar{\alpha}-\frac{1}{\bar{\alpha}}\right)+\left(\frac{\alpha}{\bar{\alpha}}-\frac{\bar{\alpha}}{\alpha}\right)\right]+\left(\bar{\alpha}-\frac{1}{\bar{\alpha}}\right)^{2}\right\}+\check{I} \cdot \frac{\left(\alpha-\frac{1}{\alpha}\right)}{\left(\bar{\alpha}-\frac{1}{\bar{\alpha}}\right)}=0 \\
& y_{1}=-\frac{\alpha A\left(1-(\alpha A \breve{I})^{2}\right)^{2}}{2} \cdot \frac{\check{I}}{\left(\alpha-\frac{1}{\alpha}\right)\left(\bar{\alpha}-\frac{1}{\bar{\alpha}}\right)} \cdot\left\{\left(\frac{\alpha}{\bar{\alpha}}-\frac{\bar{\alpha}}{\alpha}\right)\left[\left(\alpha A+\frac{1}{\alpha A}\right)\left(\bar{\alpha}-\frac{1}{\bar{\alpha}}\right)+\left(\frac{\alpha}{\bar{\alpha}}-\frac{\bar{\alpha}}{\alpha}\right)\right]+\left(\bar{\alpha}-\frac{1}{\bar{\alpha}}\right)^{2}-\left(\alpha-\frac{1}{\alpha}\right)^{2}\right\} \\
& \left(\bar{\alpha}-\frac{1}{\bar{\alpha}}\right)^{2}-\left(\alpha-\frac{1}{\alpha}\right)^{2} \equiv\left[\bar{\alpha}-\frac{1}{\bar{\alpha}}-\left(\alpha-\frac{1}{\alpha}\right)\right]\left[\bar{\alpha}-\frac{1}{\bar{\alpha}}+\left(\alpha-\frac{1}{\alpha}\right)\right] \equiv\left[\bar{\alpha}-\alpha-\left(\frac{1}{\bar{\alpha}}-\frac{1}{\alpha}\right)\right][\bar{\alpha}+\alpha- \\
& \left.\left(\frac{1}{\bar{\alpha}}+\frac{1}{\alpha}\right)\right] \equiv(\bar{\alpha}-\alpha)\left(1+\frac{1}{\bar{\alpha} \alpha}\right)(\bar{\alpha}+\alpha)\left(1-\frac{1}{\bar{\alpha} \alpha}\right) \equiv\left(\bar{\alpha}^{2}-\alpha^{2}\right)\left(1-\frac{1}{(\bar{\alpha} \alpha)^{2}}\right) \equiv\left(\frac{\bar{\alpha}^{2}-\alpha^{2}}{\bar{\alpha} \alpha}\right)\left(\bar{\alpha} \alpha-\frac{1}{\bar{\alpha} \alpha}\right) \equiv \\
& -\left(\frac{\alpha}{\bar{\alpha}}-\frac{\bar{\alpha}}{\alpha}\right)\left(\bar{\alpha} \alpha-\frac{1}{\bar{\alpha} \alpha}\right) \\
& y_{1}=-\frac{\alpha A \cdot\left(1-(\alpha A \check{I})^{2}\right)^{2}}{2} \cdot \frac{\check{I} \cdot\left(\frac{\alpha}{\bar{\alpha}}-\frac{\bar{\alpha}}{\alpha}\right)}{\left(\alpha-\frac{1}{\alpha}\right)\left(\bar{\alpha}-\frac{1}{\bar{\alpha}}\right)} \cdot\left\{\left[\left(\alpha A+\frac{1}{\alpha A}\right)\left(\bar{\alpha}-\frac{1}{\bar{\alpha}}\right)+\left(\frac{\alpha}{\bar{\alpha}}-\frac{\bar{\alpha}}{\alpha}\right)\right]-\left(\bar{\alpha} \alpha-\frac{1}{\bar{\alpha} \alpha}\right)\right\} \\
& \left(\frac{\alpha}{\bar{\alpha}}-\frac{\bar{\alpha}}{\alpha}\right)-\left(\bar{\alpha} \alpha-\frac{1}{\bar{\alpha} \alpha}\right) \equiv-\bar{\alpha} \alpha+\frac{\alpha}{\bar{\alpha}}-\frac{\bar{\alpha}}{\alpha}+\frac{1}{\bar{\alpha} \alpha} \equiv-\alpha \cdot\left(\bar{\alpha}-\frac{1}{\bar{\alpha}}\right)-\frac{1}{\alpha} \cdot\left(\bar{\alpha}-\frac{1}{\bar{\alpha}}\right) \equiv-\left(\bar{\alpha}-\frac{1}{\bar{\alpha}}\right)\left(\alpha+\frac{1}{\alpha}\right) \\
& y_{1}=-\frac{\alpha A \cdot\left(1-(\alpha A \check{I})^{2}\right)^{2}}{2} \cdot \frac{\check{I} \cdot\left(\frac{\alpha}{\bar{\alpha}}-\frac{\bar{\alpha}}{\alpha}\right)}{\left(\alpha-\frac{1}{\alpha}\right)\left(\bar{\alpha}-\frac{1}{\bar{\alpha}}\right)} \cdot\left\{\left[\left(\alpha A+\frac{1}{\alpha A}\right)\left(\bar{\alpha}-\frac{1}{\bar{\alpha}}\right)-\left(\bar{\alpha}-\frac{1}{\bar{\alpha}}\right)\left(\alpha+\frac{1}{\alpha}\right)\right]\right\} \equiv-\frac{\alpha A \cdot\left(1-(\alpha A \check{I})^{2}\right)^{2}}{2} . \\
& \frac{\check{I} \cdot\left(\frac{\alpha}{\bar{\alpha}} \bar{\alpha} \frac{\bar{\alpha}}{\alpha}\right)}{\left(\alpha-\frac{1}{\alpha}\right)} \cdot\left\{\left[\left(\alpha A+\frac{1}{\alpha A}\right)-\left(\alpha+\frac{1}{\alpha}\right)\right]\right\}
\end{aligned}
$$

$$
\begin{aligned}
& \frac{1}{\alpha A}-\frac{1}{\alpha} \equiv \frac{1}{\alpha}\left(\frac{1}{A}-1\right) \equiv \frac{1}{\alpha}\left(f+\alpha^{2} \cdot(1-f)-1\right) \equiv \frac{1}{\alpha} \cdot(1-f)\left(\alpha^{2}-1\right) \equiv(1-f)\left(\alpha-\frac{1}{\alpha}\right) \\
& \alpha(A-1) \equiv \alpha \cdot\left(\frac{1}{f+\alpha^{2} \cdot(1-f)}-1\right) \equiv \alpha \cdot \frac{1-f-\alpha^{2} \cdot(1-f)}{f+\alpha^{2} \cdot(1-f)} \equiv \alpha \cdot(1-f) \frac{1-\alpha^{2}}{f+\alpha^{2} \cdot(1-f)} \\
& \left(\alpha A+\frac{1}{\alpha A}\right)-\left(\alpha+\frac{1}{\alpha}\right) \equiv \alpha \cdot(1-f) \frac{1-\alpha^{2}}{f+\alpha^{2} \cdot(1-f)}+(1-f)\left(\alpha-\frac{1}{\alpha}\right) \equiv(1-f)\left(\alpha-\frac{1}{\alpha}\right)[1- \\
& \left.\frac{\alpha^{2}}{f+\alpha^{2} \cdot(1-f)}\right] \equiv(1-f)\left(\alpha-\frac{1}{\alpha}\right) \frac{f+\alpha^{2} \cdot(1-f)-\alpha^{2}}{f+\alpha^{2} \cdot(1-f)} \equiv(1-f)\left(\alpha-\frac{1}{\alpha}\right) \frac{f \cdot\left(1-\alpha^{2}\right)}{f+\alpha^{2} \cdot(1-f)}
\end{aligned}
$$

we finally obtain

$$
\begin{aligned}
& y_{1}=-\frac{\alpha A \cdot\left(1-(\alpha A \check{I})^{2}\right)^{2}}{2} \cdot \frac{\check{I} \cdot\left(\frac{\alpha}{\bar{\alpha}}-\frac{\bar{\alpha}}{\alpha}\right)}{f+\alpha^{2} \cdot(1-f)} \cdot(1-f) \cdot f \cdot\left(1-\alpha^{2}\right)=-\frac{f \cdot(1-f)}{2} \cdot \alpha \cdot\left(1-\alpha^{2}\right) \cdot A^{2} \cdot\left(\frac{\alpha}{\bar{\alpha}}-\frac{\bar{\alpha}}{\alpha}\right) \\
& {\left[1-(\alpha A \check{I})^{2}\right]^{2} \cdot \check{I}}
\end{aligned}
$$

Thus,

$$
y=\frac{s_{j}}{c_{o}}=y_{0}+\beta y_{1}=\alpha A \check{I} \cdot\left\{1+\frac{f \cdot(1-f)}{2} \cdot \beta \cdot\left(\alpha^{2}-1\right) \cdot A \cdot\left(\frac{\alpha}{\bar{\alpha}}-\frac{\bar{\alpha}}{\alpha}\right) \cdot\left[1-(\alpha A \check{I})^{2}\right]^{2}\right\}
$$


Remembering that $y$ is the sum of ion fluxes divided by the total ion concentration in the bulk solution, we now need to develop this into an expression for individual ion fluxes. Based on the definition of $S_{j}$ and $\mathrm{Eq}(40)$,

$j_{1} \cdot\left(\frac{1}{P_{1}}-\frac{1}{P_{2}}\right) \equiv S_{j}-\frac{\tilde{I}}{P_{2}}$

$\frac{j_{1}}{C_{0}}=\frac{y-\frac{\tilde{I}}{C_{0} P_{2}}}{\frac{1}{P_{1}}-\frac{1}{P_{2}}}=\sqrt{P_{1} P_{2}} \frac{y-\sqrt{\frac{P_{1}}{P_{2}}} \frac{\tilde{I}}{\alpha-\frac{1}{C_{0} P_{2}}}}{\alpha-\frac{1}{\alpha}}=\sqrt{P_{1} P_{2}} \cdot \frac{y-\frac{\check{I}}{\alpha}}{\alpha-\frac{1}{\alpha}}=\sqrt{P_{1} P_{2}} \cdot \frac{y_{0}-\frac{\check{I}}{\alpha}+\beta y_{1}}{\alpha-\frac{1}{\alpha}}$

In principle, we can simply substitute $\mathrm{Eq}(137)$ into $\mathrm{Eq}(139)$ to obtain an expression for $j_{1}$. However, a simpler form in terms of only permeances and ion concentrations is preferable. To obtain such an expression we note that from $\mathrm{Eq}(\mathrm{S} 127)$ that $A=\frac{2}{\left(\alpha^{2}+1\right)-2 \frac{C_{\Delta, o}}{C_{0}}\left(\alpha^{2}-1\right)}=\frac{1}{f+\alpha^{2} \cdot(1-f)^{\prime}} ; f=\frac{c_{10}}{c_{10}+c_{20}}$.

Using $\mathrm{Eq}(\mathrm{S} 126)$, one can show that

$\alpha A \check{I}=\frac{\alpha \check{I}}{f+\alpha^{2} \cdot(1-f)}=\frac{\sqrt{\frac{P_{2}}{P_{1} C_{0} \sqrt{P_{1} P_{2}}}}}{\frac{c_{1}}{c_{1}+c_{2}}+\frac{P_{2}}{P_{1}} \cdot\left(\frac{c_{2}}{c_{1}+c_{2}}\right)}=\frac{\frac{\tilde{I}}{2 P_{1}}}{c_{1}+\frac{P_{2}}{P_{1}} c_{2}}=\frac{\frac{\tilde{I}}{2}}{P_{1} c_{1}+P_{2} c_{2}}=\frac{\tilde{I}}{\tilde{I} \text { lim }}=\frac{I}{I_{\text {lim }}}$

In Eq(S140), $\tilde{I}_{l i m}=2\left(P_{1} c_{10}+P_{2} c_{20}\right)$.

Substituting from $\mathrm{Eq}(\mathrm{S} 126)$,

$y_{0}-\frac{\check{I}}{\alpha}=\alpha A \check{I}-\frac{\check{I}}{\alpha}=\check{I} \cdot\left(\alpha A-\frac{1}{\alpha}\right)$

Moreover, based on Eq(127),

$\alpha A-\frac{1}{\alpha}=\frac{1}{\frac{f}{\alpha}+\alpha \cdot(1-f)}-\frac{1}{\alpha}=\frac{\alpha-\frac{f}{\alpha}-\alpha \cdot(1-f)}{f+\alpha^{2} \cdot(1-f)}=\frac{f \cdot\left(\alpha-\frac{1}{\alpha}\right)}{f+\alpha^{2} \cdot(1-f)}$.

Substituting Eqs(S136, S141,S142) into Eq(S139) and rearranging gives

$\frac{j_{1}}{C_{0} \sqrt{P_{1} P_{2}}}=\frac{y_{0}-\frac{\breve{I}}{\alpha}+\beta y_{1}}{\alpha-\frac{1}{\alpha}}=\check{I} \cdot \frac{f}{f+\alpha^{2} \cdot(1-f)}+\frac{\beta y_{1}}{\alpha-\frac{1}{\alpha}}=\frac{f \cdot \check{I}}{f+\alpha^{2} \cdot(1-f)}+\alpha A \check{I} \cdot\left\{\frac{f \cdot(1-f)}{2} \cdot \beta \cdot \alpha A \cdot\left(\frac{\alpha}{\bar{\alpha}}-\frac{\bar{\alpha}}{\alpha}\right)\right.$.
$\left.\left[1-(\alpha A \check{I})^{2}\right]^{2}\right\}$

Further substituting $\mathrm{Eq}(\mathrm{S} 140)$ and the definitions of $\frac{\alpha}{\bar{\alpha}^{\prime}}, \frac{\bar{\alpha}}{\alpha}$, and $\beta \equiv \frac{L}{c_{X} / C_{0}} \cdot \sqrt{\frac{P_{1} P_{2}}{\bar{D}_{1} \bar{D}_{2}}}$ while noting that $\beta\left(\frac{\alpha}{\bar{\alpha}}-\frac{\bar{\alpha}}{\alpha}\right)=\frac{L}{c_{X} / C_{0}}\left(\frac{P_{2}}{\bar{D}_{2}}-\frac{P_{1}}{\bar{D}_{1}}\right)$ leads to

$\frac{j_{1}}{C_{0} \sqrt{P_{1} P_{2}}}=\frac{f \cdot \check{I}}{f+\alpha^{2} \cdot(1-f)}+\frac{L}{c_{X} / C_{0}} \cdot\left(\frac{\tilde{I}}{\tilde{I}_{\text {lim }}}\right) \frac{f \cdot(1-f)}{2} \cdot \alpha A\left(\frac{P_{2}}{\overline{\bar{D}}_{2}}-\frac{P_{1}}{\bar{D}_{1}}\right)\left(1-\left(\frac{\tilde{I}}{\tilde{I}_{l i m}}\right)^{2}\right)^{2}$

Finally, solving for $j_{1}$, noting that $\tilde{I}=\check{I} C_{0} \sqrt{P_{1} P_{2}} ; f=\frac{c_{10}}{c_{10}+c_{20}} ; C_{0}=2 c_{10}+2 c_{20}$, using Eq(S140) and rearranging yields 


$$
\begin{aligned}
& j_{1}=\frac{f \cdot \tilde{I}}{f+\alpha^{2} \cdot(1-f)}+C_{0} \sqrt{P_{1} P_{2}} \frac{L}{c_{X} / C_{0}} \cdot\left(\frac{I}{I_{\text {lim }}}\right) \frac{f \cdot(1-f)}{2} \cdot \alpha A\left(\frac{P_{2}}{\bar{D}_{2}}-\frac{P_{1}}{\bar{D}_{1}}\right)\left(1-\left(\frac{I}{I_{\text {lim }}}\right)^{2}\right)^{2}=\frac{P_{1} c_{10}}{P_{1} c_{10}+P_{2} c_{20}} \cdot \tilde{I}+ \\
& \sqrt{P_{1} P_{2}} \frac{L}{c_{X}} \cdot\left(\frac{I}{I_{\text {lim }}}\right) 2 c_{10} c_{20} \cdot \alpha A\left(\frac{P_{2}}{\bar{D}_{2}}-\frac{P_{1}}{\bar{D}_{1}}\right)\left(1-\left(\frac{I}{I_{\text {lim }}}\right)^{2}\right)^{2}=\frac{P_{1} c_{10}}{P_{1} c_{10}+P_{2} c_{20}} \cdot \tilde{I}+\sqrt{P_{1} P_{2}}\left(\frac{L}{c_{X}}\right) \cdot\left(\frac{I}{I_{\text {lim }}}\right) \cdot \\
& \frac{2 c_{10} c_{20}}{\frac{f}{\alpha}+\alpha \cdot(1-f)}\left(\frac{P_{2}}{\bar{D}_{2}}-\frac{P_{1}}{\bar{D}_{1}}\right)\left(1-\left(\frac{I}{I_{\text {lim }}}\right)^{2}\right)^{2}=\frac{P_{1} c_{10}}{P_{1} c_{10}+P_{2} c_{20}} \cdot \tilde{I}+\left(\frac{L}{c_{X} / C_{0}}\right) \cdot\left(\frac{I}{I_{\text {lim }}}\right) \cdot \frac{c_{10} c_{20} P_{1} P_{2}}{P_{1} c_{10}+P_{2} c_{20}}\left(\frac{P_{2}}{\bar{D}_{2}}-\right. \\
& \left.\frac{P_{1}}{\bar{D}_{1}}\right)\left(1-\left(\frac{I}{I_{\text {lim }}}\right)^{2}\right)^{2}=2 P_{1} c_{10}\left(\frac{I}{I_{\text {lim }}}\right) \cdot\left\{1-\left(\frac{L}{2 c_{X} / C_{0}}\right) \cdot \frac{c_{20} P_{2}}{P_{1} c_{10}+P_{2} c_{20}}\left(\frac{P_{1}}{\bar{D}_{1}}-\frac{P_{2}}{\bar{D}_{2}}\right)\left(1-\left(\frac{I}{I_{\text {lim }}}\right)^{2}\right)^{2}\right\} \text { (S145) }
\end{aligned}
$$

S12. Derivation of Eq(46) for the potential drop during current passage through an ion-exchange membrane: First-order correction to the limiting case of constant electrochemical potentials across an ion-exchange membrane

This derivation begins with Eq(27), which we repeat below for convenience.

$\varphi(-1-L)-\varphi(1+L)=2\left[\frac{L \bar{S}_{j}}{c_{X}}+\ln \left(\frac{C_{0}+S_{j}}{C_{0}-S_{j}}\right)\right]$

We derived $\mathrm{Eq}(27)$ in Section S3. Remembering that $y=S_{j} / C_{0}$

$\varphi(-1-L)-\varphi(1+L)=2\left[\frac{L \bar{S}_{j}}{c_{X}}+\ln \left(\frac{1+y}{1-y}\right)\right]$

As shown previously in $\mathrm{Eq}(\mathrm{S} 113)$,

$\frac{L \bar{S}_{j}}{c_{X}}=\beta \cdot\left[y \cdot\left(\frac{\bar{\alpha}-\frac{1}{\bar{\alpha}}}{\alpha-\frac{1}{\alpha}}\right)+\check{I} \cdot\left(\frac{\alpha-\frac{\bar{\alpha}}{\alpha}-\frac{\bar{\alpha}}{\alpha}}{\alpha-\frac{1}{\alpha}}\right)\right]$

Note that in $\mathrm{Eq}(\mathrm{S} 113) y$ is multiplied by $\beta$, which is small, so we can neglect the correction and replace $y$ with $y_{0}=\alpha A \check{I}(\mathrm{Eq}(\mathrm{S} 126))$.

With appropriate substitutions of $\operatorname{Eqs}(\mathrm{S} 113, \mathrm{~S} 126)$, and using the first-order correction $y=y_{0}+\beta y_{1}$, we obtain

$\varphi(-1-L)-\varphi(1+L) \approx 2 \beta \cdot\left[\alpha A \check{I} \cdot\left(\frac{\bar{\alpha}-\frac{1}{\bar{\alpha}}}{\alpha-\frac{1}{\alpha}}\right)+\check{I} \cdot\left(\frac{\frac{\alpha}{\bar{\alpha}}-\frac{\bar{\alpha}}{\alpha}}{\alpha-\frac{1}{\alpha}}\right)\right]+2\left[\ln \left(\frac{1+y_{0}}{1-y_{0}}\right)+\ln \left(\frac{1+\frac{\beta y_{1}}{1+y_{0}}}{1-\frac{\beta y_{1}}{1-y_{0}}}\right)\right]$

Noting that for small $x, \ln (1+x)=x$ and $\ln (1-x)=-x$

$\varphi(-1-L)-\varphi(1+L) \approx 2 \beta \cdot\left[\alpha A \check{I} \cdot\left(\frac{\bar{\alpha}-\frac{1}{\bar{\alpha}}}{\alpha-\frac{1}{\alpha}}\right)+\check{I} \cdot\left(\frac{\alpha}{\overline{\bar{\alpha}}-\frac{\bar{\alpha}}{\alpha}} \frac{1}{\alpha-\frac{1}{\alpha}}\right)\right]+2\left[\ln \left(\frac{1+y_{0}}{1-y_{0}}\right)+\beta y_{1}\left(\frac{1}{1+y_{0}}+\frac{1}{1-y_{0}}\right)\right](\mathrm{S} 14$

Identical transformations give 
$\varphi(-1-L)-\varphi(1+L) \approx 2 \beta \cdot\left[\alpha A \check{I} \cdot\left(\frac{\bar{\alpha}-\frac{1}{\bar{\alpha}}}{\alpha-\frac{1}{\alpha}}\right)+\check{I} \cdot\left(\frac{\frac{\alpha}{\bar{\alpha}}-\frac{\bar{\alpha}}{\alpha}}{\alpha-\frac{1}{\alpha}}\right)\right]+2\left[\ln \left(\frac{1+y_{0}}{1-y_{0}}\right)+\frac{2 \beta y_{1}}{1-y_{0}^{2}}\right]=2 \ln \left(\frac{1+y_{0}}{1-y_{0}}\right)+2 \beta \cdot$

$\left[\alpha A \check{I} \cdot\left(\frac{\bar{\alpha}-\frac{1}{\bar{\alpha}}}{\alpha-\frac{1}{\alpha}}\right)+\check{I} \cdot\left(\frac{\frac{\alpha}{\bar{\alpha}}-\frac{\bar{\alpha}}{\alpha}}{\alpha-\frac{1}{\alpha}}\right)+\frac{2 y_{1}}{1-y_{0}^{2}}\right]$

Based on Eqs(S126,S140), $y_{0}=\frac{\tilde{I}}{\tilde{I}_{\text {lim }}}=\frac{I}{I_{\text {lim }}}$, Additionally, with appropriate identical transformations,

$\alpha A \cdot\left(\frac{\bar{\alpha}-\frac{1}{\bar{\alpha}}}{\alpha-\frac{1}{\alpha}}\right)+\left(\frac{\frac{\alpha}{\bar{\alpha}}-\frac{\bar{\alpha}}{\alpha}}{\alpha-\frac{1}{\alpha}}\right)=\frac{\alpha\left[\frac{\bar{\alpha}}{\alpha} f+\frac{\alpha}{\bar{\alpha}}(1-f)\right]}{f+\alpha^{2}(1-f)}=\frac{\frac{\bar{\alpha}}{\alpha} \cdot f+\frac{\alpha}{\bar{\alpha}} \cdot(1-f)}{\frac{f}{\alpha}+\alpha \cdot(1-f)}$. Substituting these expressions into Eq(S149), along with $\mathrm{Eq}(\mathrm{S} 136)$ and $\mathrm{Eq}(\mathrm{S} 140)$ gives

$\varphi(-1-L)-\varphi(1+L) \approx 2 \ln \left(\frac{1+I / I_{l i m}}{1-I / I_{l i m}}\right)+2 \beta \check{I} \cdot\left[\frac{\frac{\bar{\alpha}}{\alpha} \cdot f+\frac{\alpha}{\bar{\alpha}} \cdot(1-f)}{\frac{f}{\alpha}+\alpha \cdot(1-f)}+f \cdot(1-f) \cdot \alpha \cdot\left(\alpha^{2}-1\right) \cdot A^{2} \cdot\left(\frac{\alpha}{\bar{\alpha}}-\frac{\bar{\alpha}}{\alpha}\right)\right.$.

$\left.\left(1-\left(\frac{I}{I_{l i m}}\right)^{2}\right)\right]$

Noting that $\alpha A=\frac{1}{\frac{f}{\alpha}+\alpha \cdot(1-f)}, \mathrm{Eq}(\mathrm{S} 150)$ becomes

$\varphi(-1-L)-\varphi(1+L)=2 \ln \left(\frac{1+I / I_{\text {lim }}}{1-I / I_{\text {lim }}}\right)+2 \beta \alpha A \check{I} \cdot\left[\left(\frac{\bar{\alpha}}{\alpha} \cdot f+\frac{\alpha}{\bar{\alpha}} \cdot(1-f)\right)+f \cdot(1-f) \cdot\left(\alpha^{2}-1\right) \cdot A \cdot\right.$

$\left.\left(\frac{\alpha}{\bar{\alpha}}-\frac{\bar{\alpha}}{\alpha}\right) \cdot\left(1-\left(\frac{I}{I_{\text {lim }}}\right)^{2}\right)\right]$

Using the following identities

$\beta \equiv \frac{L}{c_{X} / C_{0}} \sqrt{\frac{P_{1} P_{2}}{\bar{D}_{1} \bar{D}_{2}}} ; \frac{\bar{\alpha}}{\alpha} \equiv \sqrt{\frac{\overline{D_{2} P_{1}}}{\bar{D}_{1} P_{2}}} ; \beta \frac{\bar{\alpha}}{\alpha}=\frac{L}{c_{X} / C_{0}} \sqrt{\frac{P_{1} P_{2}}{\bar{D}_{1} \bar{D}_{2}}} \sqrt{\frac{\bar{D}_{2} P_{1}}{\bar{D}_{1} P_{2}}}=\frac{L}{c_{X} / C_{0}} \frac{P_{1}}{\bar{D}_{1}} ; \beta \frac{\alpha}{\bar{\alpha}} \equiv \frac{L}{c_{X} / C_{0}} \sqrt{\frac{P_{1} P_{2}}{\bar{D}_{1} \bar{D}_{2}}} \sqrt{\frac{\bar{D}_{1} P_{2}}{\bar{D}_{2} P_{1}}} \equiv \frac{L}{c_{X} / C_{0}} \frac{P_{2}}{\bar{D}_{2}}$

$\varphi(-1-L)-\varphi(1+L)=2 \ln \left(\frac{1+I / I_{\text {lim }}}{1-I / I_{\text {lim }}}\right)+\frac{2 L C_{0}}{c_{X}} \cdot\left(\frac{I}{I_{\text {lim }}}\right) \cdot\left[\left(\frac{P_{1}}{\bar{D}_{1}} \cdot f+\frac{P_{2}}{\bar{D}_{2}} \cdot(1-f)\right)+f \cdot(1-f) \cdot\right.$

$\left.\left(\alpha-\frac{1}{\alpha}\right) \cdot \alpha A \cdot\left(\frac{P_{2}}{\bar{D}_{2}}-\frac{P_{1}}{\bar{D}_{1}}\right) \cdot\left(1-\left(\frac{I}{I_{l i m}}\right)^{2}\right)\right]$

Noting that $C_{0} f \cdot(1-f) \cdot\left(\alpha-\frac{1}{\alpha}\right) \cdot \alpha A=\frac{2 c_{10} c_{20}}{c_{10}+c_{20}} \frac{P_{2}-P_{1}}{f P_{1}+(1-f) P_{2}}=\frac{2 c_{10} c_{20} \cdot\left(P_{2}-P_{1}\right)}{P_{1} c_{10}+P_{2} c_{20}}$ and using identical transformations leads to

$\varphi(-1-L)-\varphi(1+L)=2\left\{\ln \left(\frac{1+I / I \text { lim }}{1-I / I_{\text {lim }}}\right)+\frac{2 L}{c_{X}} \cdot\left(\frac{I}{I_{\text {lim }}}\right)\left[\frac{P_{1} c_{10}}{\bar{D}_{1}}+\frac{P_{2} c_{20}}{\bar{D}_{2}}+\frac{c_{10} c_{20} \cdot\left(P_{1}-P_{2}\right)}{P_{1} c_{10}+P_{2} c_{20}} \cdot\left(\frac{P_{1}}{\bar{D}_{1}}-\frac{P_{2}}{\bar{D}_{2}}\right)\right.\right.$. $\left.\left.\left(1-\left(\frac{I}{I_{\text {lim }}}\right)^{2}\right)\right]\right\}$ 


\section{S13. Numerical simulations of bi-ionic potentials and fluxes}

Nernst-Planck Equation. The model for ion transport includes diffusion and electromigration in the two boundary layers, ion partitioning at the boundary layer/membrane interfaces, and diffusion and electromigration in the membrane. We employ the Nernst-Planck equation (S154) to describe the fluxes, $j_{i}$, of specific ions in the boundary layer.

$-\frac{j_{i}}{D_{i}}=\frac{d c_{i}}{d x}+z_{i} c_{i} \frac{d \varphi}{d x}$

As previously described, $D_{i}$ is the diffusion coefficient in solution, $c_{i}$ is the real ion concentration, which depends on the coordinate $x, z_{i}$ is the ion charge, and $\varphi$ is dimensionless (in F/RT units) real electrical potential. In the ion-exchange membrane, equation (S155) describes the fluxes.

$-\frac{j_{i}}{\bar{D}_{i}}=\frac{d \bar{c}_{i}}{d \bar{x}}+z_{i} \bar{c}_{i} \frac{d \bar{\varphi}}{d \bar{x}}$

The overbars denote that the specific variables apply to the ion-exchange membrane. The concentrations and electrical potential are real, rather than virtual, quantities because we use a diffusion coefficient rather than a permeability coefficient. Thus, we also need to determine partitioning at the interface of the boundary later and the membrane. In this case, we employ the Donnan model to describe ion partitioning.

The Donnan model. In equilibrium partitioning of an ion between two phases, the ion's electrochemical potentials should be equal in the two phases. Considering membrane, $M$, and solution, $S$, phases, this gives equation (S156),

$\bar{\mu}_{i}^{M}=\bar{\mu}_{i}^{S}$

where $\bar{\mu}_{i}^{M}$ and $\bar{\mu}_{i}^{S}$ are the electrochemical potentials in the membrane and solution, respectively. Substituting for the electrochemical potentials leads to

$\mu_{i}^{o M}+R T \ln a_{i}^{M}+z_{i} F \phi^{M}=\mu_{i}^{o S}+R T \ln a_{i}^{S}+z_{i} F \phi^{S}$

In these equations, $\mu_{i}^{o}$ is the standard state chemical potential, $a_{i}$ is the ion activity, and $\phi$ is the electrical potential for the denoted phase. Further, $z_{i}$ is the ion charge, $R$ is the gas constant and $T$ is temperature. The Donnan model assumes that $\mu_{i}^{O M}=\mu_{i}^{O S}$ and that activity coefficients are unity so activities equal concentrations. These assumptions lead to

$\phi^{M}-\phi^{S}=\frac{R T}{z_{i} F} \ln \frac{C_{i}^{S}}{C_{i}^{M}}$

For a system with three ions, we can equate the potential differences for all three ions.

$\frac{R T}{z_{1} F} \ln \frac{C_{1}^{S}}{C_{1}^{M}}=\frac{R T}{z_{2} F} \ln \frac{C_{2}^{S}}{C_{2}^{M}}=\frac{R T}{z_{3} F} \ln \frac{C_{3}^{S}}{C_{3}^{M}}$ or $\left(\frac{C_{1}^{M}}{C_{1}^{S}}\right)^{1 / z_{1}}=\left(\frac{C_{2}^{M}}{C_{2}^{S}}\right)^{1 / z_{2}}=\left(\frac{C_{3}^{M}}{C_{3}^{S}}\right)^{1 / z_{3}}$

We define a partition coefficient $\Gamma_{i}$ 
$\Gamma_{i}=\frac{C_{i}^{M}}{C_{i}^{S}}$

Substituting this definition into equation (S159) yields

$\Gamma_{2}=\frac{C_{2}^{M}}{C_{2}^{S}}=\Gamma_{1}^{\frac{z_{2}}{z_{1}}}$ and $\Gamma_{3}=\frac{C_{3}^{M}}{C_{3}^{S}}=\Gamma_{1}^{\frac{z_{3}}{z_{1}}}$

For a three-ion system, the electrical neutrality condition inside the membrane is

$z_{1} C_{1}^{M}+z_{2} C_{2}^{M}+z_{3} C_{3}^{M}+z_{x} C_{x}^{M}=0$.

Using equations (S161) to define the concentrations in the membrane, equation (S162) becomes

$z_{1} C_{1}^{S} \Gamma_{1}+z_{2} C_{2}^{S} \Gamma_{1}^{\frac{z_{2}}{z_{1}}}+z_{3} C_{3}^{S} \Gamma_{1}^{\frac{z_{3}}{z_{1}}}+z_{x} C_{x}^{M}=0$

Knowing the ion concentrations in solution, for a mixture of $\mathrm{KCl}$ and $\mathrm{LiCl}$ this is a quadratic equation that one can solve for $\Gamma_{1}$. Subsequently equation (S161) allows calculation of other ion partition coefficients and the concentrations of each ion in the membrane.

Numerical Procedures. Equations (S154 and S155) are systems of three equations (one equation for each ion). We assume that the ion-exchange membrane is homogeneous $\left(\frac{d c_{x}}{d \bar{x}}=0\right)$. With the assumption of electroneutrality $\left(\sum_{i} z_{i} c_{i}=0\right.$ and $\left.\sum_{i} z_{i} \overline{c_{l}}=c_{x}\right)$, one can transform equations (S154 and S155) into:

$\frac{d c_{i}}{d x}=-\frac{j_{i}}{D_{i}}+z_{i} c_{i} \frac{\sum_{i} z_{i} \frac{j_{i}}{D_{i}}}{\sum_{i} z_{i}^{2} c_{i}}$

$\frac{d \overline{c_{l}}}{d \bar{x}}=-\frac{j_{i}}{\bar{D}_{i}}+z_{i} \overline{\bar{c}_{l}} \frac{\sum_{i} z_{i} \frac{j_{i}}{\sum_{i} z_{i}^{2} \bar{c}_{i}}}{\sum_{i}}$

We assumed a thermodynamic equilibrium established at the boundary layer/membrane interface and employed the Donnan model to relate $c_{i}$ and $\overline{c_{l}}$ at the interfaces. We treated equations (S164 and S165) as initial value problems by specifying one set of the bulk concentrations $c_{i}(-1-L)$ as initial conditions. By inputting two ion fluxes (the third flux is specified by the zero-current condition, $\sum_{i} z_{i} j_{i}=0$ ), we solved equations (S164 and S165) using a differential equation solver that is based on an explicit Runge-Kutta formula to get the ion concentration profiles in the boundary layers and in the membrane. In the MATLAB program, we performed iterations on the two ion fluxes until the other set of bulk concentrations $c_{i}(1+L)$, obtained from the solver, converged with the ones we specified.

\section{S14. Numerical simulations of current-induced concentrations polarization}

The procedures for simulating current-induced concentrations polarization are largely like the ones we employed in section S13. However, the zero-current condition $\left(\sum_{i} z_{i} j_{i}=0\right)$ is replaced with equation (S166):

$F \sum_{i} z_{i} j_{i}=I$

$F$ is the Faraday's constant and $I$ is the current density. We employed the same Nernst-Planck equations to describe ion transport and the Donnan model to describe ion partitioning. The numerical procedures 
are the same as well. We treated equations (S164 and S165) as initial value problems by specifying one set of the bulk concentrations $c_{i}(-1-L)$ as initial conditions. By specifying current density and inputting two ion fluxes (the third flux is specified by equation (S166)), we solved equations (S164 and S165) using a differential equation solver that is based on an explicit Runge-Kutta formula to get the ion concentration profiles in the boundary layers and in the membrane. In the MATLAB program, we performed iterations on the two ion fluxes until the other set of bulk concentrations $c_{i}(1+L)$, obtained from the solver, converged with the ones we specified. We provide the MATLAB program here.

\section{MATLAB code}

This is a sample program that solves the concentration profiles of $\mathrm{K}^{+}, \mathrm{Li}^{+}$, and $\mathrm{Cl}^{-}$. The current density is $5.5 \mathrm{~A} / \mathrm{dm}^{2}$. The fixed charge density of the cation-exchange membrane is $1 \mathrm{M}$ (negative in the program to account for the negative fixed charges). Boundary layers are each $100 \mu \mathrm{m}$ thick while the membrane is $50 \mu \mathrm{m}$ thick. We employed literature infinite dilution values for diffusion coefficients of ions in the boundary layer and assumed different extents of reduction of diffusion coefficients in the cation exchange membrane. The bulk concentrations are $0.1 \mathrm{M} \mathrm{KCl}$ and $0.1 \mathrm{M} \mathrm{LiCl}$ in the mixture. All parameters can be easily changed in the program. However, one should input reasonable initial guesses to facilitate the iteration process.

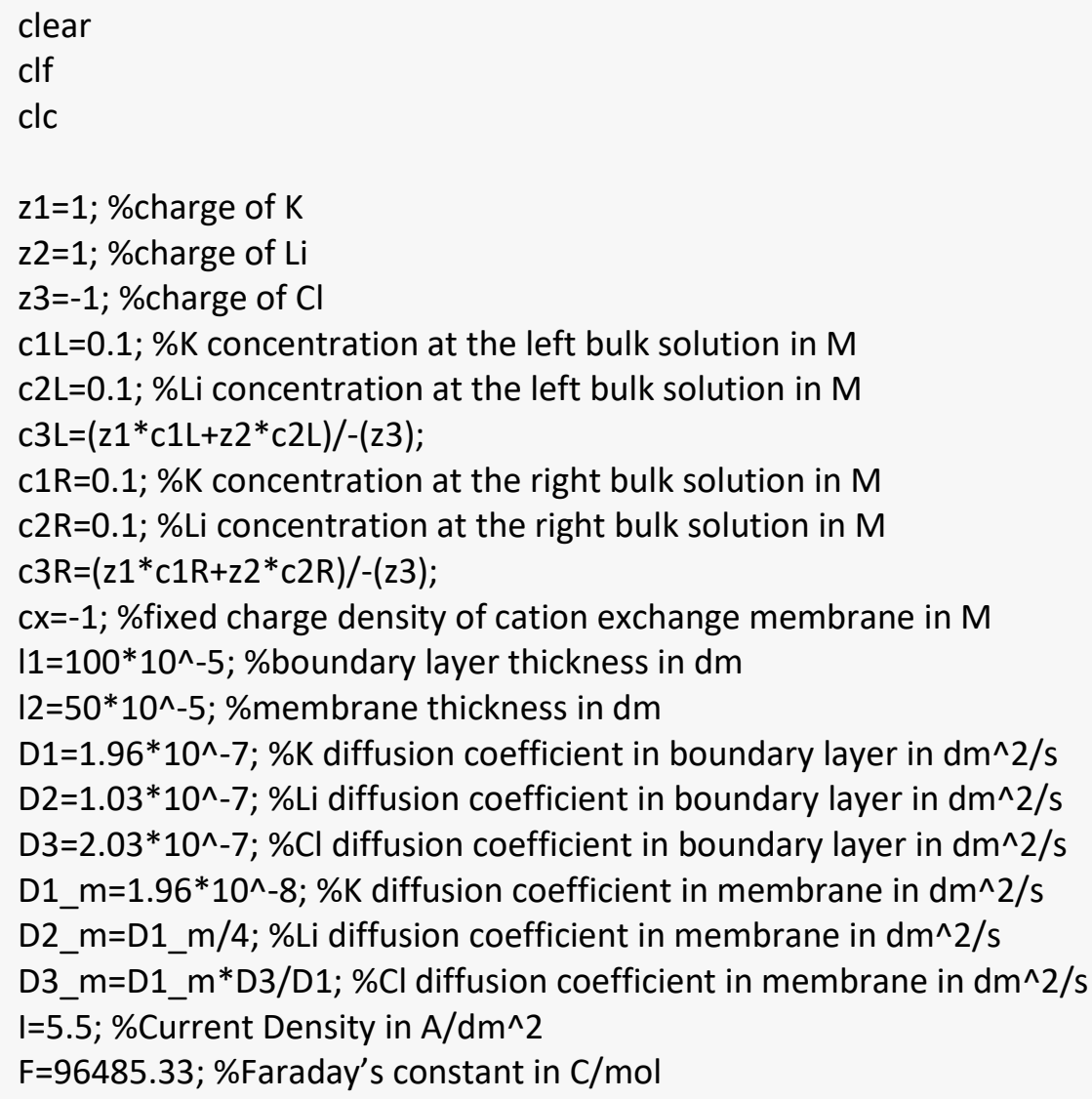


options = optimset('TolX',1e-18,'TolFun',1e-18,'MaxFunEvals',1e13,'Maxlter',1e13);

sol=fminsearch(@(j) funcSolve $(j, z 1, z 2, z 3$,

$\left.c 1 L, c 2 L, c 3 L, c 1 R, c 2 R, c 3 R, c x, I 1, I 2, D 1, D 2, D 3, D 1 \_m, D 2 \_m, D 3 \_m, I, F\right),[j 1, j 2]$,options);

j1=sol(1);

$\mathrm{j} 2=\operatorname{sol}(2)$;

function [res] = funcSolve (j, z1,z2,z3,c1L,c2L,c3L,c1R,c2R,c3R,cx,I1,I2,D1,D2,D3,D1_m,D2_m,D3_m,I,F)

$\mathrm{j} 1=\mathrm{j}(1)$;

$\mathrm{j} 2=\mathrm{j}(2)$;

$j 3=(I-z 1 * F * j 1-z 2 * F * j 2) / z 3 / F$;

[t1,c1]=ode45(@(t1,c1) func1(c1, D1,D2,D3,z1,z2,z3,j1,j2,j3,I1), [0 1], [c1L,c2L,c3L]);

c1_int1=c1(end,1);

c2_int1=c1(end,2);

c3_int1=c1(end,3);

p1 $=\left[z 1^{*} c 1 \_\right.$int $1+z 2 * c 2 \_$int 1 cx z3*c3_int1];

$\mathrm{r} 1=\operatorname{roots}(\mathrm{p} 1)$;

gamma1=r1(imag(r1)==0 \& $r 1>=0)$;

co1=gamma1*c1_int1;

co2 $=\left(\right.$ gamma1^ $\left.\left.{ }^{\wedge}(\mathrm{z} 2 / \mathrm{z} 1)\right)\right)^{*} \mathrm{c} 2$ int1;

co3 $=(\text { gamma1^ }(z 3 / z 1))^{*}$ c3_int1;

[t2,c2]=ode45(@(t2,c2) func1(c2, D1_m,D2_m,D3_m,z1,z2,z3,j1,j2,j3,I2), [0 1], [co1,co2,co3]);

ce1=c2 (end, 1 );

ce2 $=$ c2 (end, 2 );

ce3=c2 (end, 3 );

p2 $=[z 3 *$ ce3 0 z1*ce1+z2*ce2];

$\mathrm{r} 2=\operatorname{roots}(\mathrm{p} 2)$;

gamma2=r2(imag(r2)==0 \& $r 2>=0)$;

c1_int2 = ce1/gamma2;

c2_int2 $=$ ce2/gamma2^(z2/z1);

c3_int2 = ce3/gamma2^(z3/z1);

[t3,c3]=ode45(@(t3,c3) func1(c3, D1,D2,D3,z1,z2,z3,j1,j2,j3,I1), [0 1], [c1_int2,c2_int2,c3_int2]);

c1R_out=c3(end,1);

c2R_out=c3(end,2); 
c3R_out=c3(end,3);

res $=(\text { c1R_out } / c 1 R-1)^{\wedge} 2+\left(c 2 R \_o u t / c 2 R-1\right)^{\wedge} 2+\left(c 3 R \_o u t / c 3 R-1\right)^{\wedge} 2 ;$

function dc = func1 $(c, D 1, D 2, D 3, z 1, z 2, z 3, j 1, j 2, j 3, I)$

$\mathrm{dc}=[-\mathrm{j} 1 * \mathrm{I} / \mathrm{D} 1+(\mathrm{z} 1 * \mathrm{c}(1) * \mathrm{*}((\mathrm{z} 1 * \mathrm{j} 1 / \mathrm{D} 1)+(\mathrm{z} 2 * \mathrm{j} 2 / \mathrm{D} 2)+(\mathrm{z} 3 * \mathrm{j} 3 / \mathrm{D} 3))) /(\mathrm{z} 1 * \mathrm{z} 1 * \mathrm{c}(1)+\mathrm{z} 2 * \mathrm{z} 2 * \mathrm{c}(2)+\mathrm{z} 3 * \mathrm{z} 3 * \mathrm{c}(3))$; $-j 2 * 1 / D 2+(z 2 * c(2) * 1 *((z 1 * j 1 / D 1)+(z 2 * j 2 / D 2)+(z 3 * j 3 / D 3))) /(z 1 * z 1 * c(1)+z 2 * z 2 * c(2)+z 3 * z 3 * c(3)) ;$ $-j 3 * 1 / D 3+(z 3 * c(3) * 1 *((z 1 * j 1 / D 1)+(z 2 * j 2 / D 2)+(z 3 * j 3 / D 3))) /(z 1 * z 1 * c(1)+z 2 * z 2 * c(2)+z 3 * z 3 * c(3))]$; end

end

\section{S15. Numerical simulations for modelling concentration profiles above a bipolar ion-exchange patch}

To calculate the salt-concentration profiles in the two-dimensional system with a patch of a perfectly selective ion exchanger, we used Comsol Multiphysics 4.2 software. Ion fluxes in the electrolyte solution above the wall with the IEX patch were modeled using the extended Nernst-Planck Equation module, which generally accounts for diffusion, electro-migration and convective components of ion flows. However, we set the fluid flow rate to zero, as we assumed negligible convective flow. The diffusion coefficients of the ions of a 1:1 electrolyte were assumed to be $D_{1}=D_{2}=2 \cdot 10^{-9} \mathrm{~m}^{2} / \mathrm{s}$, and their electrical mobility was defined as $u_{1,2}=\frac{D_{1,2}}{R T}$. We assumed the patch width to be equal to $20 \mu \mathrm{m}$ positioning it on the $x$-axis from $x=-10 \mu \mathrm{m}$ to $x=+10 \mu \mathrm{m}$.

The electrolyte domain was chosen in the form of a hemi-cylinder located above the wall with the center in the middle of the patch and with a radius of $100 \mu \mathrm{m}$. For graphing purposes, additional sections were made at the heights of $2 \mu \mathrm{m}, 4 \mu \mathrm{m}$, and $6 \mu \mathrm{m}$. The mesh was generated automatically with the largest element size being $0.5 \mu \mathrm{m}$ and the smallest being $0.001 \mu \mathrm{m}$. A mesh layer with increased resolution was added at the wall for improving the accuracy.

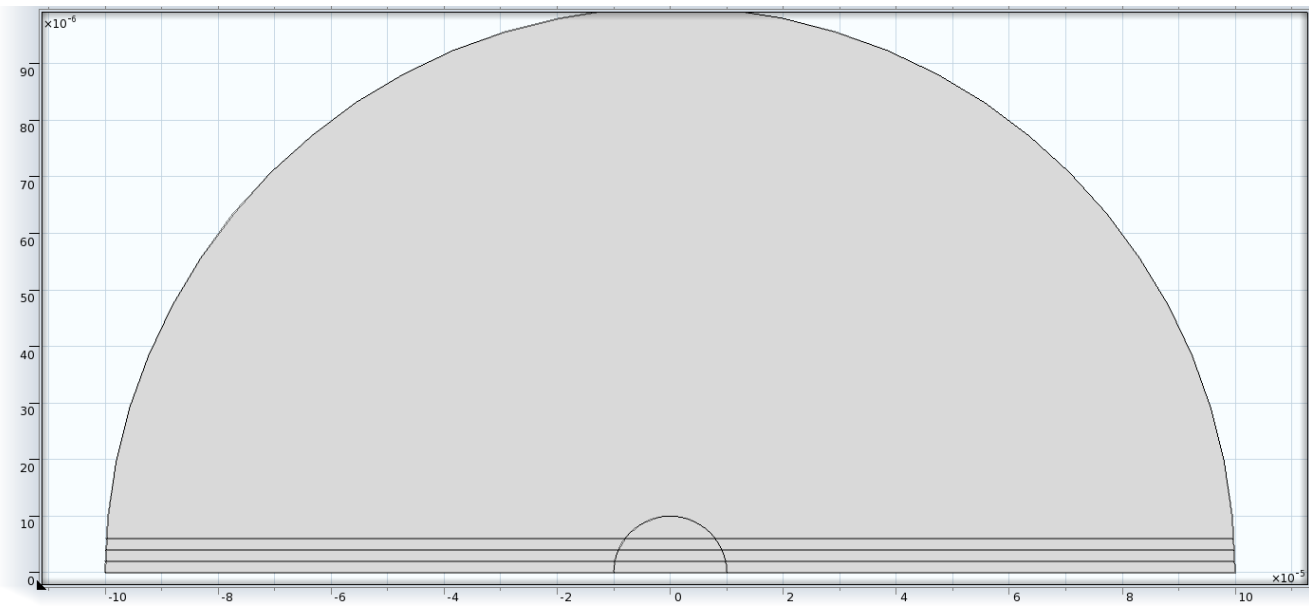

Computational domain 
Since the problem involves finding two quantities, salt concentration and electrostatic potential, on each of the sections of the boundary it is necessary to set two boundary conditions. On the semi-circular boundary of the domain we set a constant electrolyte concentration equal to the bulk concentration (assumed to be $1 \mathrm{mM}$ ) and an electric potential that corresponds to an uniform electric field of a given strength: $\varphi=-U_{0} x$.

At the wall outside the patch, the boundary conditions reflect the absence of flux of both ions normal to the surface. Obviously, these conditions automatically mean zero electrical current through the wall. For the coions the zero normal flux condition applies to the patch region, too. The second boundary condition at the patch reflects its very high permeability to the counterions so their electrochemical potential along the patch does not change:

$\mu_{1}=\mu_{1}^{0}+R T \ln C+F Z_{1} \varphi$ is a constant, so $R T \ln C+F Z_{1} \varphi$ is also a constant.

We iterated on the value of $R T \ln C+F Z_{1} \varphi$ so that the net normal flux of counter-ions (and electric current) through the patch was zero. At the same time, the counterions were allowed to "enter" the patch from the solution in one part of it, but had to "exit" it from another. Thus, the condition for choosing $\mu_{1}$ reflects the fact that the patch is insulated from external sources of ions. 